\title{
Surface brightness fluctuation distances for dwarf elliptical galaxies in the Fornax cluster ${ }^{\star}$
}

\author{
H. Jerjen ${ }^{\star \star}$ \\ Research School of Astronomy and Astrophysics, The Australian National University, Mt Stromlo Observatory, Cotter Road, \\ Weston ACT 2611, Australia
}

Received 20 September 2002 / Accepted 30 October 2002

\begin{abstract}
We have obtained deep $B$ and $R$-band CCD images of eight dwarf elliptical (dE) galaxies in the Fornax cluster using the FORS1 instrument at the VLT in service mode under excellent atmospheric conditions. A total of 92 fields distributed over the central regions of the galaxies have been analysed to measure local $(B-R)_{0}$ colours and $R$-band surface brightness fluctuation (SBF) magnitudes $\bar{m}_{R}$. Within a galaxy the observed correlation of $(B-R)_{0}$ with $\bar{m}_{R}^{0}$ follows closely the predicted slope of the colour-fluctuation luminosity relation for composite single-burst, mainly old, metal-poor stellar populations. This allows to determine the distances of the dEs from simple offset measurements to a typically $9 \%$ accuracy. The distance distribution of these genuine cluster dwarfs centers at a mean distance of $(m-M)_{0}=31.54 \pm 0.07 \mathrm{mag}$, or $20.3 \pm 0.7 \mathrm{Mpc}$, a value that is in best agreement with previous SBF work on Fornax early-type giants and thus represents a robust estimate of the distance to the Fornax cluster core. The application of the bootstrap resampling technique on the distance data further reveals a cluster depth of $\sigma_{\text {int }}=1.4_{-0.8}^{+0.5} \mathrm{Mpc}$. We take this preliminary result as a confirmation of the compact appearance of Fornax in the projection on the sky although the cluster might be slightly more elongated along the line of sight. Combining the newly derived cluster distance with the cosmological velocity of Fornax of $1324 \pm 41 \mathrm{~km} \mathrm{~s}^{-1}$ gives a Hubble constant of $H_{0}=65 \pm 4 \mathrm{~km} \mathrm{~s}^{-1} \mathrm{Mpc}^{-1}$. This value is consistent at the $95 \%$ confidence level with both the most recent result from the team that favours a long distance scale and the final value adopted by the HST "Key Project" team in their work for the Hubble constant. Finally, we explore the possibility to determine rough metallicities of our cluster dEs from their $(B-R)_{0}$ colours via Worthey's stellar population synthesis models. The median metallicities are found in the range from -1.5 to -1 with a concentration around $[\mathrm{Fe} / \mathrm{H}]=-1$. A comparison with spectral line indices results available for three galaxies shows good agreement. Moreover, the derived metallicities place the bright Fornax dEs on the extension of the metallicity-luminosity relation defined by the low luminous Local Group dEs which provides additional support for the $(B-R)_{0}$ colour as a useful metallicity estimator. The data further suggest an age range between 10 and $12 \mathrm{Gyr}$ for the Fornax dwarfs.
\end{abstract}

Key words. galaxies: clusters: individual: Fornax - galaxies: distances and redshifts - galaxies: dwarf galaxies: elliptical and lenticular, cD - galaxies: individual: IC1919 - galaxies: stellar content

\section{Introduction}

The galaxy cluster in Fornax plays a fundamental role in many astronomical fields. A good understanding of its 3-dimensional structure and the knowledge of an accurate cluster distance are key issues for cosmology, galaxy and cluster formation and evolution, and in particular the cosmological distance scale. The Fornax cluster is the second nearest galaxy cluster after Virgo from the Local Group hosting about 350 galaxy members (Ferguson 1989; Phillipps et al. 2001). Due to its compactness and structural simplicity, Fornax appears as a less complex and thus potentially better understandable galaxy aggregate than the Virgo Cluster. But Fornax nevertheless presents its own difficulties for a precise distance measurement. The galaxies in Fornax are essentially concentrated in two clumps (see Fig. 1).

* Based on observations collected at the European Southern Observatory (ESO 68.A-0176).

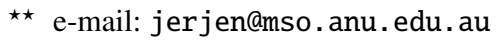

The major component is rich in early-type galaxies and centered around the giant elliptical NGC 1399. Approximately three degrees to the southwest lies a much smaller subcluster that is dominated by the peculiar Sa galaxy NGC 1316 and composed of mainly late-type galaxies. Dynamical evidence for substructure in Fornax was found just recently (Drinkwater et al. 2001). The concentration around NGC 1316 has a slightly higher $\left(\approx 100 \mathrm{~km} \mathrm{~s}^{-1}\right)$ mean velocity suggesting an infall toward the cluster core from the near side. On the other hand, the Cepheid distances to three spiral galaxies in the Fornax region (Silbermann et al. 1999; Prosser et al. 1999; Mould et al. 2000) give the impression of a line-of-sight extension at the far side of the cluster.

The observe spread in Cepheid distances prompted Mould et al. to suggest that the mean of $21.7 \mathrm{Mpc}$ (distance corrected for metallicity effects) to be adopted as the true Fornax cluster distance. However, this is unlikely a secure value given the tendency of late-type galaxies to avoid cluster cores. A more 


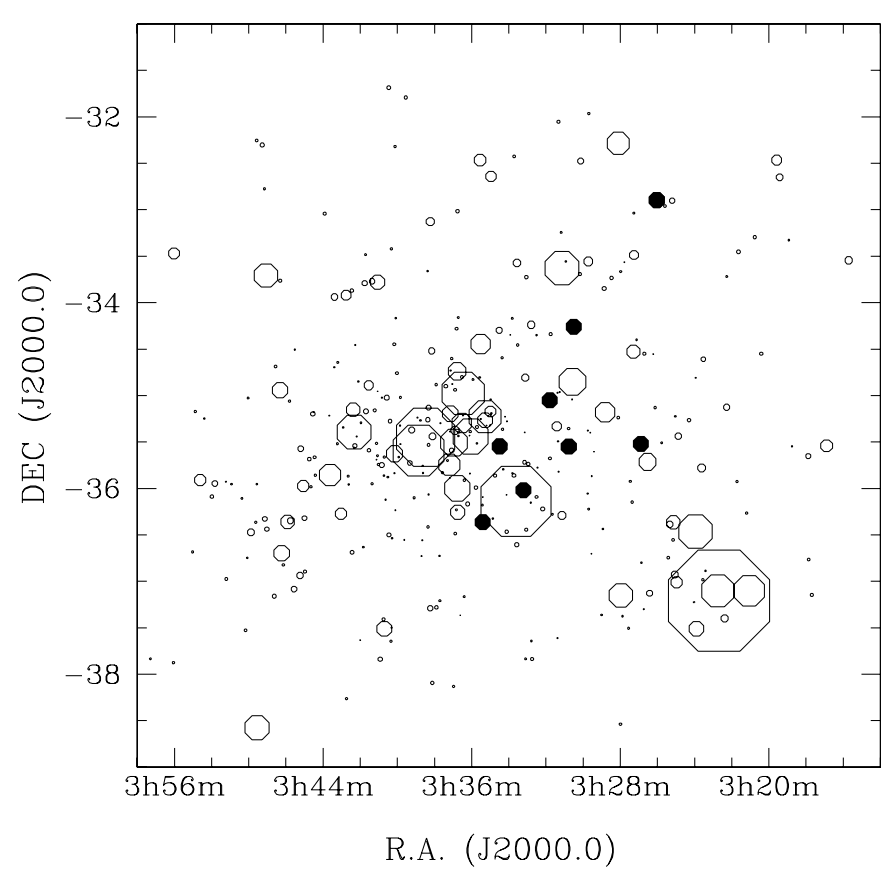

Fig. 1. A map of the galaxies in the central region of the Fornax cluster produced from the Fornax Cluster Catalog (Ferguson 1989). The size of each symbol is proportional to the galaxy luminosity. Filled circles indicate the location of the dwarf galaxies observed in the present study. The three brightest Fornax galaxies NGC 1316, NGC 1365, and NGC 1399 are located at $03 h 22 m 41.7 s,-37 d 12 m 30 s, 03 h 33 m 36.4 s$, $-36 d 08 m 25 s$, and $03 h 38 m 29.3 s,-35 d 27 m 01 s$, respectively.

robust result is awaited from observations of genuine cluster galaxies. From the scientific point of view the best choice are the low-surface brightness dwarf elliptical (dE) galaxies. Firstly, their existence is well confined to the high density environment (Binggeli et al. 1987; Ferguson \& Binggeli 1994; Jerjen \& Dressler 1997). Secondly, dwarf ellipticals are the only galaxy type that also exist in large numbers in galaxy clusters ( $\sim 250$ in the case of the Fornax cluster, Ferguson 1989) so that uncertainties due to individual distance errors and possible substructural depth effects can be reduced statistically. The combination of these two properties naturally identifies dEs as ideal objects to measure distances to all sorts of galaxy aggregates.

Distance informations for a large sample of cluster dEs would clearly offer a unique opportunity to study the Fornax cluster core in unprecedented details. But unfortunately it is notoriously difficult to measure accurate distances to dEs. Empirical scaling laws like the effective surface brightness-luminosity relation (Binggeli \& Cameron 1991) or the shape parameter-luminosity relation (Jerjen \& Binggeli 1997; Binggeli \& Jerjen 1998) have considerable scatter $(\sigma \sim$ $0.8 \mathrm{mag}$ ) rendering them as fairly modest distance indicators. The alternative approach would be the use of the tip magnitude of the red giant branch (TRGB). But the requirement of precise photometry of individual stars in a galaxy at the distance of the Fornax cluster is costly and time consuming, restricting the practicability essentially to few systems. The involved effort was demonstrated by Harris et al. (1998) who spent $32000 \mathrm{~s}$ integration time with the Hubble Space Telescope to estimate the TRGB distance of one single dE (IC3388) in the Virgo cluster.

Clearly, a more practical and less expensive method is needed if full advantage shall be taken over the large number of available dwarf ellipticals. For that purpose, Jerjen et al. (1998, 2000,2001 ) have tested the $R$-band surface brightness fluctuations (SBFs) as a new distance indicator for dEs in the distance range between $2-10 \mathrm{Mpc}$. The theoretical framework of the method was developed by Tonry \& Schneider (1988) with the aim to measure distances to high surface brightness giant ellipticals. However, Jerjen and collaborators demonstrated that the method works equally well for low surface brightness systems. Technically, the method is based on the discrete sampling of a stellar population in a galaxy with a CCD detector and the resulting Poisson fluctuations in the number of stars within a resolution element.

The first results on the empirical calibration of the $R$-band SBF distance indicator for dEs (Jerjen et al. 2001) were encouraging. Good agreement was also found between observations and theoretical predictions for mainly old, metal-poor stellar populations from stellar synthesis models (Worthey 1994) suggesting an accuracy of the method at the $\approx 10-15$ percent level. This is significantly better than any other comparable distance indicator for early-type dwarfs available to date. The high accuracy and the modest requirement in observing time and telescope size opens up the all new possibility to measure distances to a large number of dEs in a simple way. Two hours of imaging in two photometric bands $(B$ and $R$ ) at a ground-based $2.5 \mathrm{~m}$ class telescope under good seeing conditions is sufficient to estimate the distance of a $10 \mathrm{Mpc}$ dwarf elliptical galaxy (Jerjen et al. 2001).

Looking beyond the $10 \mathrm{Mpc}$ boundary, the most obvious and exciting applications of the SBF method for dEs is the mapping of the Fornax and Virgo clusters in three dimensions and the spatial identification of the gravitational hot spots in these galaxy aggregates. To explore this possibility we have been awarded observing time at the ESO-VLT to study the distance distributions of 16 Virgo dEs and $25 \mathrm{dEs}$ in the Fornax cluster. While the results of the Virgo dEs will be published in a forthcoming paper (Jerjen et al. 2003), we can report in the present paper on the SBF results for a first set of eight earlytype dwarfs in the Fornax cluster for which data acquisition is complete. These Fornax dEs were chosen from the Fornax Cluster Catalog (Ferguson 1989) because of their appearance as promising candidates for a SBF analysis. The criteria were: (i) morphological type $\mathrm{dE}(, \mathrm{N})$ or $\mathrm{dSO}(, \mathrm{N})$, (ii) large diameter at an isophote of $26.5 \mathrm{BJ}_{J} \mathrm{arcsec}^{-2}$, (iii) a low visual flattening, and (iv) a high mean surface brightness.

The projected spatial distribution of the selected dwarfs relative to the other cluster galaxies is shown in Fig. 1 and their basic properties are listed in Table 1: Cols. 1, 2 - Fornax cluster Catalog (FCC) number and morphological type (Ferguson 1989). For simplicity we will use the term "dE" for all dwarfs in the following. Columns 3, 4 - Right ascension and declination for the epoch J2000, Col. 5 - Integrated apparent $B$-magnitude (Ferguson 1989), Col. 6 - Heliocentric velocity (Held \& Mould 1994; Da Costa et al. 1998; Stein 2002), Cols. 7, 8 - Galactic absorption in $B$ and $R$-bands (Schlegel et al. 1998). 
Table 1. Basic properties of the observed early-type dwarf galaxies in the Fornax cluster.

\begin{tabular}{cccccccc}
\hline \hline FCC & Type & $\begin{array}{c}\text { RA } \\
(\mathrm{J} 2000.0)\end{array}$ & $\begin{array}{c}\text { Decl. } \\
(\mathrm{J} 2000.0)\end{array}$ & $\begin{array}{c}B_{T} \\
(\mathrm{mag})\end{array}$ & $\begin{array}{c}v_{\odot} \\
\mathrm{km} \mathrm{s}^{-1}\end{array}$ & $\begin{array}{c}A_{B} \\
\mathrm{mag}\end{array}$ & $\begin{array}{c}A_{R} \\
\mathrm{mag}\end{array}$ \\
$(1)$ & $(2)$ & $(3)$ & $(4)$ & $(5)$ & $(6)$ & $(7)$ & $(8)$ \\
\hline 043 & $\mathrm{dS0,N}$ & $03 \mathrm{~h} 26 \mathrm{~m} 02.2 \mathrm{~s}$ & $-32 \mathrm{~d} 53 \mathrm{~m} 41 \mathrm{~s}$ & 13.5 & 1334 & 0.062 & 0.038 \\
050 & $\mathrm{dE} 0, \mathrm{~N}$ & $03 \mathrm{~h} 26 \mathrm{~m} 54.1 \mathrm{~s}$ & $-35 \mathrm{~d} 31 \mathrm{~m} 04 \mathrm{~s}$ & 16.6 & - & 0.042 & 0.026 \\
082 & $\mathrm{dE} 1, \mathrm{~N}$ & $03 \mathrm{~h} 30 \mathrm{~m} 30.4 \mathrm{~s}$ & $-34 \mathrm{~d} 15 \mathrm{~m} 31 \mathrm{~s}$ & 16.4 & - & 0.063 & 0.039 \\
085 & $\mathrm{dE} 0, \mathrm{~N}$ & $03 \mathrm{~h} 30 \mathrm{~m} 46.1 \mathrm{~s}$ & $-35 \mathrm{~d} 32 \mathrm{~m} 53 \mathrm{~s}$ & 16.3 & 1509 & 0.060 & 0.037 \\
100 & $\mathrm{dE}, \mathrm{N}$ & $03 \mathrm{~h} 31 \mathrm{~m} 47.6 \mathrm{~s}$ & $-35 \mathrm{~d} 03 \mathrm{~m} 07 \mathrm{~s}$ & 15.5 & 1660 & 0.062 & 0.038 \\
116 & $\mathrm{dE} 1, \mathrm{~N}$ & $03 \mathrm{~h} 33 \mathrm{~m} 12.8 \mathrm{~s}$ & $-36 \mathrm{~d} 01 \mathrm{~m} 05 \mathrm{~s}$ & 16.1 & 1635 & 0.097 & 0.060 \\
136 & $\mathrm{dE} 2, \mathrm{~N}$ & $03 \mathrm{~h} 34 \mathrm{~m} 29.5 \mathrm{~s}$ & $-35 \mathrm{~d} 32 \mathrm{~m} 47 \mathrm{~s}$ & 14.8 & 1205 & 0.069 & 0.043 \\
150 & $\mathrm{dE} 4, \mathrm{~N}$ & $03 \mathrm{~h} 35 \mathrm{~m} 24.1 \mathrm{~s}$ & $-36 \mathrm{~d} 21 \mathrm{~m} 51 \mathrm{~s}$ & 15.7 & 2014 & 0.060 & 0.037 \\
\hline
\end{tabular}

Table 2. Observing log of the imaging data taken in service mode.

\begin{tabular}{|c|c|c|c|c|c|c|c|c|c|c|}
\hline $\begin{array}{l}\text { FCC } \\
(1)\end{array}$ & $\begin{array}{l}\text { Filter } \\
\text { (2) }\end{array}$ & $\begin{array}{l}\text { UT Date } \\
\text { (3) }\end{array}$ & $\begin{array}{c}\text { Mean } \\
\text { Airmass } \\
(4)\end{array}$ & $\begin{array}{l}\text { Exp. time } \\
\text { (s) } \\
\text { (5) }\end{array}$ & $\begin{array}{c}F W H M \\
(\operatorname{arcsec}) \\
(6)\end{array}$ & $\begin{array}{l}\text { Filter } \\
(7)\end{array}$ & $\begin{array}{c}\text { UT Date } \\
\text { (8) }\end{array}$ & $\begin{array}{c}\text { Mean } \\
\text { Airmass } \\
(9)\end{array}$ & $\begin{array}{c}\text { Exp. time } \\
\text { (s) } \\
\text { (10) }\end{array}$ & $\begin{array}{c}F W H M \\
(\operatorname{arcsec}) \\
(11)\end{array}$ \\
\hline 043 & $B$ & $2001-10-22$ & 1.01 & $3 \times 500$ & 0.54 & $R$ & $2001-10-22$ & 1.02 & $3 \times 700$ & 0.45 \\
\hline 050 & $B$ & $2001-10-24$ & 1.15 & $3 \times 500$ & 0.80 & $R$ & 2001-10-24 & 1.09 & $3 \times 700$ & 0.63 \\
\hline 082 & B & $2001-10-24$ & 1.22 & $3 \times 500$ & 0.73 & $R$ & 2001-11-09 & 1.02 & $3 \times 700$ & 0.49 \\
\hline 082 & $B$ & $2001-11-18$ & 1.22 & $3 \times 500$ & 0.85 & $R$ & 2002-01-19 & 1.03 & $3 \times 700$ & 0.45 \\
\hline 085 & $B$ & $2001-11-18$ & 1.14 & $3 \times 500$ & 0.70 & $R$ & 2001-11-18 & 1.08 & $3 \times 700$ & 0.53 \\
\hline 100 & $B$ & $2001-12-07$ & 1.02 & $3 \times 500$ & 0.85 & $R$ & 2001-11-18 & 1.02 & $3 \times 700$ & 0.49 \\
\hline 116 & $B$ & $2001-11-19$ & 1.05 & $3 \times 500$ & 0.66 & $R$ & 2002-01-19 & 1.08 & $3 \times 700$ & 0.45 \\
\hline 136 & $B$ & 2001-11-19 & 1.04 & $3 \times 500$ & 0.57 & $R$ & 2002-01-19 & 1.16 & $3 \times 700$ & 0.45 \\
\hline 150 & $B$ & $2001-11-19$ & 1.02 & $3 \times 500$ & 0.60 & $R$ & 2002-03-15 & 1.59 & $3 \times 700$ & 0.65 \\
\hline
\end{tabular}

The structure of the paper is as follows. The observations and data reduction are presented in Sect. 2. We describe the SBF analysis and how the fluctuation signals were measured in Sect. 3. In Sect. 4 we interpret the results based on comparison with stellar population models and we determine the SBF distances of the galaxies. The mean distance to the Fornax cluster as defined by the dwarfs is discussed in Sect. 5 and a preliminary value for the line-of-sight cluster depth is calculated. The value of the Hubble constant is derived in Sect. 6. We estimate the typical age and metallicity of the dEs from $(B-R)_{0}$ colours and test the $[\mathrm{Fe} / \mathrm{H}]$-luminosity relation for early-type dwarfs in Sect. 7. A summary and conclusions can be found in Sect. 8.

\section{Observations and reduction}

Deep images of the eight dwarf galaxies were obtained in the Bessell $B$ and $R$ passbands using the FORS1 (FOcal Reducer/Low dispersion Spectrograph) at UT3 of the Very Large Telescope at ESO Paranal Observatory in service mode during dark time periods in the semester 68: October 2001March 2002. The FORS1 detector is a thinned and antireflection coated Tektronix CCD with $2048 \times 2048$ pixels. By default, service observations are taken in standard resolution mode and with a high gain. The pixel scale of 0.2 pixel $^{-1}$ yields a field of view of $6.8 \times 6.8$. The CCD was read out in the fourport mode, i.e. four amplifiers read out one quarter of the CCD each. Target galaxies were positioned close to the center of one of the four quadrants so that the other three remained "empty" and thus could be used later as night flats. For each galaxy a set of three exposures of $500 \mathrm{~s}$ in the $B$-band and $700 \mathrm{~s}$ in the $R$-band were secured with slightly different pointings. For FCC082 a second set of images was obtained yielding a total of six frames in each filter. The observing log is given in Table 2.

The FORS data were reduced in the standard way with IRAF $^{1}$. We subtracted the bias level from each CCD quadrant using the overscan regions and a masterbias that was generated from the template bias frames taken every observing night. Twilight sky exposures were median-combined after level normalisation to generate twilight masterflats for each filter. In the $R$-band, the twilight masterflat was further improved using the nightflats generated from the "empty" regions on the science frames. After flatfield correction the relative sky brightness variation of the $B R$ science frames was $0.1 \%$ or better across the CCD area.

All CCD data were acquired on nights with photometric sky quality. In service mode, a series of photometric standard stars (Landolt 1992) are observed in various passbands under different airmasses every night. Based on these frequent observations, the VLT Quality Control and Trending Services

${ }^{1}$ IRAF is distributed by the National Optical Astronomy Observatories, which is operated by the Association of Universities for Research in Astronomy, Inc., under contract with the National Science Foundation. 

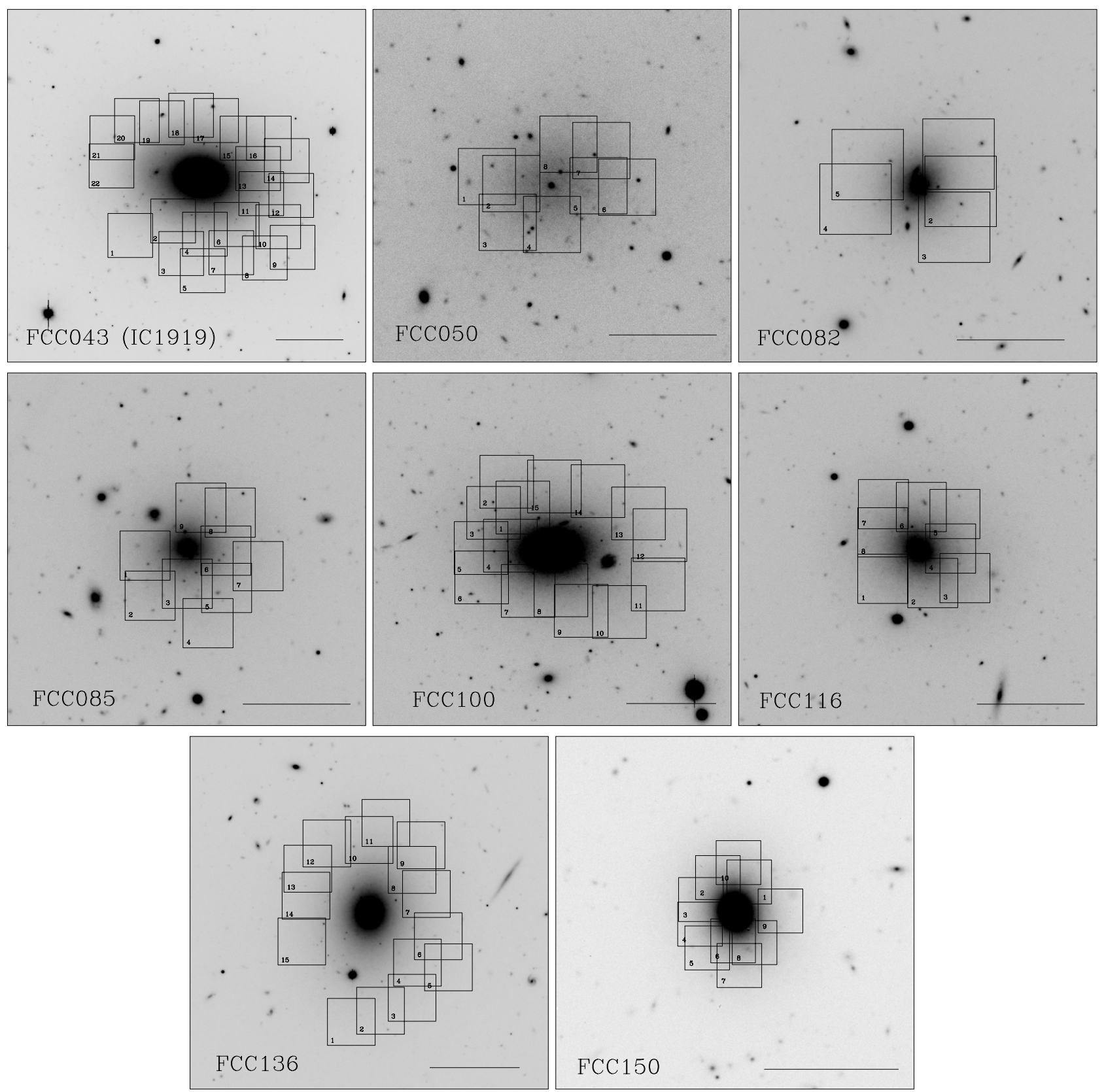

Fig. 2. VLT images of the eight sample galaxies produced by combining three $700 \mathrm{~s}$ exposures taken with FORS1 through the $R$-band filter. The numbers and boundaries of the analysed SBF fields (see text) are indicated. The bar on the images corresponds to 30 arcsec. North is at the top, east to the left.

(Hanuschik \& Silva 2002) at Garching regularly report among others on the FORS1 photometric zero points of the standard broad-band filters. Furthermore, trends for the colour terms and the extinction coefficients at Paranal are calculated. Taking advantage of this service for the photometric calibration of our CCD images we adopted the following values provided by ESO that were valid for the period of our observations:

$k_{B}=0.27, \quad k_{R}=0.13, \quad c_{(B-R)}=-0.066$.

Based on these extinction coefficients and colour term, the photometric zero points in $B$ and $R$ (see Table 3 ) were determined for each night from our own set of standard stars observations. The derived formal photometric accuracy was better than 0.03 mag in the $B$-band and 0.02 mag in the $R$-band, respectively.

In a next step, all images of a particular galaxy were registered by matching the positions of $\sim 50$ reference stars using the starfind, xyxymatch and imalign commands. The sky background level was conservatively estimated by fitting a constant to selected star-free areas distributed uniformly over the CCD area but well away from the galaxy. The sky-subtracted images of a galaxy taken in the same passband were afterwards cleaned from cosmic rays with crreject and co-added with imcombine. Finally, The master images were flux calibrated. Figure 2 shows the galaxy images resulting from the combined $R$-band data. 
Table 3. Photometric zero points of the observing nights.

\begin{tabular}{ccc}
\hline \hline UT Date & $\mathrm{ZP}(B)$ & $\mathrm{ZP}(R)$ \\
\hline $2001-10-22$ & $27.208 \pm 0.020$ & $27.483 \pm 0.011$ \\
$2001-10-24$ & $27.171 \pm 0.026$ & $27.472 \pm 0.018$ \\
$2001-11-09$ & - & $27.441 \pm 0.011$ \\
$2001-11-18$ & $27.192 \pm 0.011$ & $27.466 \pm 0.011$ \\
$2001-11-19$ & $27.210 \pm 0.015$ & - \\
$2001-11-20$ & $27.182 \pm 0.011$ & - \\
$2001-12-07$ & $27.145 \pm 0.016$ & $27.471 \pm 0.009$ \\
$2001-12-11$ & $27.215 \pm 0.011$ & - \\
$2002-01-12$ & $27.155 \pm 0.008$ & - \\
$2002-01-15$ & $27.139 \pm 0.020$ & - \\
$2002-01-19$ & - & $27.460 \pm 0.008$ \\
$2002-03-15$ & - & $27.395 \pm 0.009$ \\
\hline
\end{tabular}

\section{SBF measurements}

To prepare the $R$-band galaxy image for the SBF analysis we have to model the overall light distribution. For that purpose, we cleaned the image from any foreground stars, globular clusters, and background galaxies using procedures that follow the recipes of Jerjen et al. (2000; 2001). All point sources and extended objects brighter than the cutoff magnitude of $m_{\mathrm{c}}=27.0 \mathrm{mag}(\approx 1000$ ADUs $)$ were automatically identified using DAOPHOT II (Stetson 1987). Unwanted objects well away from the galaxy were patched with plain sky. If an object happened to interfere with the galaxy it was replaced with an adjacent uncontaminated patch from the same surface brightness range. The cleaned galaxy image was then modeled using an isophote fitting routine that allows the center, ellipticity, and position angle to vary. The best $2 \mathrm{D}$-model was subtracted from the original master image and the residual image divided by the square root of the model for noise normalization.

Any non-radial irregularities in the light distribution of dE galaxies such as dust features or even spirals (Jerjen et al. 2000; Barazza et al. 2002) are technical difficult to model and remain visible in the residual image. They indicate significant internal extinction and the possible presence of younger stars. Due to the nature of these irregularities, the effect on the measured surface brightness fluctuations would be unpredictable. These parts of a galaxy, for example the central region of FCC043, must be avoided. Only regions where the model follows closely the galaxy light distribution were used for the SBF analysis.

The largest possible number of slightly overlapping square subimages (hereafter SBF fields) were then defined on the fluctuation image within the $23.5 \mathrm{R}$ mag $\operatorname{arcsec}^{-2}$ isophotal limit thereby avoiding areas with numerous previously identified disturbing sources. The size of the SBF fields was chosen between $50 \times 50$ and $100 \times 100$ pixels depending on the apparent diameter of the galaxy. The few contaminating pixels of a SBF field were manually replaced with randomly selected patches on the fluctuation image lying outside of the field and in the same surface brightness range of the galaxy. The number of patched pixels was less than $5 \%$ of the total SBF field area in all cases. In total we defined 92 SBF fields in our eight sample galaxies. Their sizes and distribution over the galaxy's surface are shown in Fig. 2. Assuming an average seeing of $\approx 0.5$ arcsec, a field carries the SBF signal from 350 to 1400 independent points.

All SBF fields were Fourier transformed and the azimuthally averaged power spectra calculated. From isolated bright stars on the master image we determined the point spread function (PSF) profile. We then fitted a linear combination of the flux normalized and exposure time weighted PSF power spectrum and a constant at the observed galaxy power spectrum $\operatorname{PS}(k)=P_{0} \mathrm{PS}_{\mathrm{Star}}(k)+P_{1}$, demanding a least squares minimisation. Data points at low spatial frequencies $(k \leq 5)$ were omitted as they are likely affected by imperfect galaxy model subtraction. Figures 3 to 6 show the power spectrum of each SBF field with the best fitting analytic function indicated as solid line. Tables 4 and 5 summarize the quantities measured in the SBF analysis: Col. 1 - SBF field number and galaxy name, Col. 2 - pixel size of the SBF field, Col. 3 - magnitude $m_{1}$ of a star yielding $1 \mathrm{ADU}$ per second on the CCD, Col. 4 - mean galaxy surface brightness within the SBF field in ADU, Col. 5 sky brightness in ADU, Col. 6 - exposure time normalized amplitude $P_{0}$ of the best least squares fit at wave number $k=0$ with fitting error in brackets, Col. 7 - the scale-free white noise component $P_{1}$ in the power spectrum, indicating the ratio of sky to mean galaxy surface brightness within the SBF field.

Some fraction of the measured power $P_{0}(\mathrm{Col} .6)$ is not due to unresolved stars in our dwarf galaxies but to distant galaxies fainter than the cutoff magnitude $m_{\mathrm{c}}=27 R \mathrm{mag}$. To estimate the contribution of these background galaxies we made use of a formula that was given in Jensen et al. (1998) and adjusted for the $R$-band by Jerjen et al. (2001):

$P_{\mathrm{BG}}=\frac{p^{2}}{(0.8-\gamma) \ln 10} 10^{0.8\left(m_{1}-m_{\mathrm{c}}\right)-\gamma\left(29.38+R-K-m_{\mathrm{c}}\right)}$,

where $p$ is the CCD pixel size in arcsec and $\gamma=0.3$ the slope of the power-law number distribution for background galaxies in the $K$-band (Cowie et al. 1994). Assuming a typical galaxy colour of $(R-K)=2.25$ (de Jong 1996) we computed $P_{\mathrm{BG}}$ and determined the signal-to-noise $S / N=\left(P_{0}-P_{\mathrm{BG}}\right) /\left(P_{1}+P_{\mathrm{BG}}\right)$ as well as the relative contribution to the signal $P_{\mathrm{BG}} / P_{0}$ for each individual SBF field. Both numbers are listed in Cols. 8 and 9 of Tables 4 and 5. The $S / N$ ranges from 3.3 to 11.4 with a median of 6.6 whereas the contribution from unresolved galaxies was minimal at the 1-6 percent level in our SBF fields.

Another potentially significant source of fluctuations is a rich globular cluster (GC) system in a target galaxy. While this is an important issue for luminous giant ellipticals the expected number of GCs in our nucleated dwarf ellipticals is quite small. The GC frequency $\left(S_{N}\right)$-luminosity relation for dE,Ns studied in the Fornax and Virgo clusters (Miller et al. 1998) predicts $\approx 40 \mathrm{GCs}$ (assuming $S_{N}=2$ ) for our brightest dwarf FCC043 and $\approx 10 \mathrm{GCs}$ (assuming $S_{N}=10$ ) for the faintest dwarf FCC050. All GCs would be brighter than our cutoff luminosity $\left(M_{\mathrm{c}} \approx-4.5 R \mathrm{mag}\right)$ and thus be excised during the image cleaning process. Therefore, no further corrections were applied to the measured SBF power.

Finally, we calculated the stellar fluctuation magnitude $\bar{m}_{R}$ with the formula $\bar{m}_{R}=m_{1}-2.5 \log \left(P_{0}-P_{\mathrm{BG}}\right)$ and measured the $(B-R)$ colour for each $\mathrm{SBF}$ field from the cleaned $B$ 


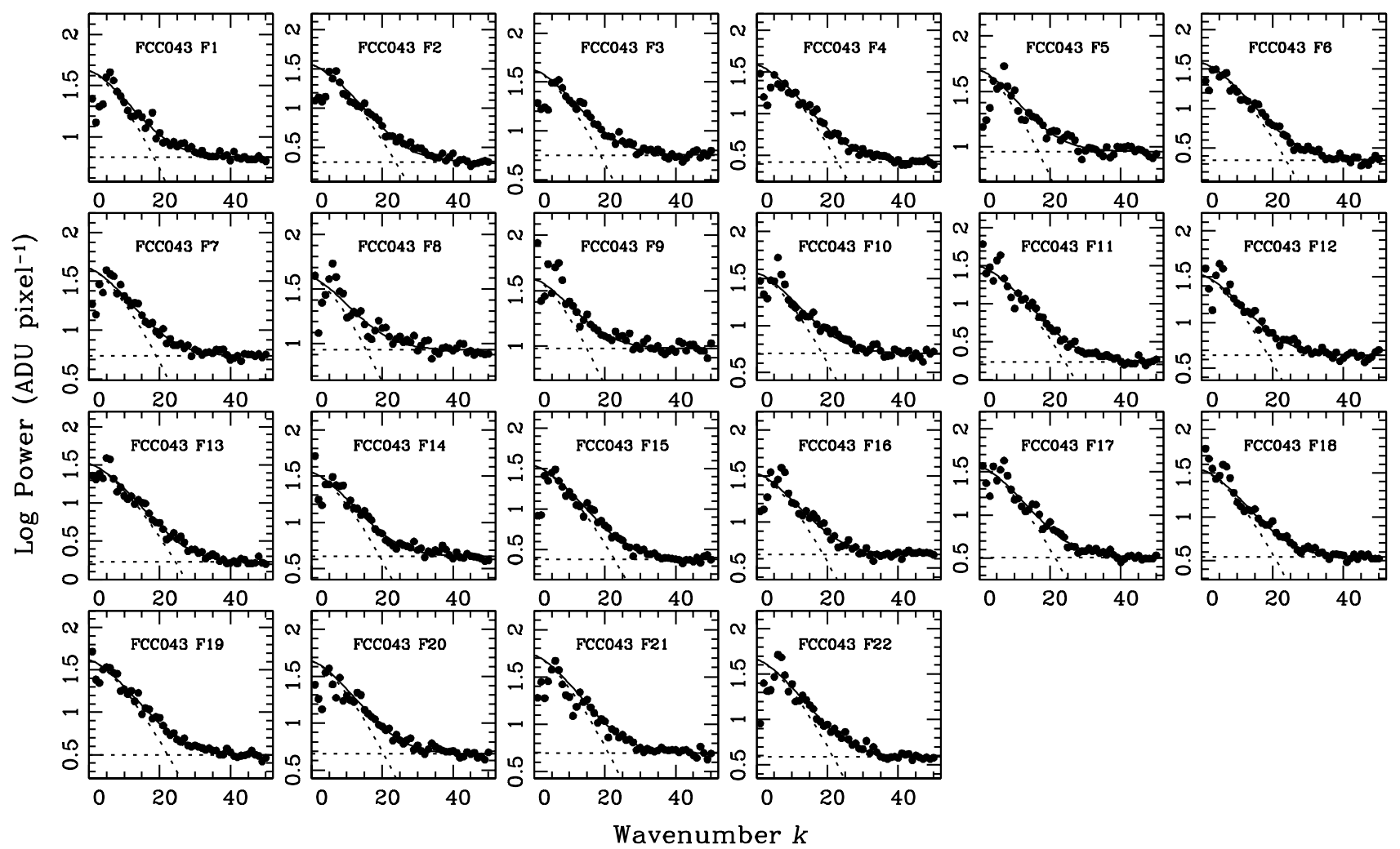

Fig. 3. For each dwarf galaxies a number of subimages were defined in the high surface brightness component that are suitable for a SBF analysis. We show here the power spectra of all fields analysed in the galaxy FCC043. The "F" numbers correspond to the SBF field numbers given in Fig. 2. The observations (crosses) are well fitted by the sum (solid line) of a scaled version of the power spectrum of the PSF and a constant (dashed lines).

and $R$ galaxy master images. Both quantities were corrected for foreground extinction using the IRAS/DIRBE maps of dust IR emission (Schlegel et al. 1998). The results are listed in Cols. 3 and 4 of Tables 6 and 7.

The power spectrum fitting error is between 2 and $17 \%$. Other sources of minor errors are the PSF normalization ( 2\%), the shape variation of the stellar PSF over the CCD area $(1-2 \%)$ and the uncertainty in the photometric calibration (0.03 mag in $B, 0.02 \mathrm{mag}$ in $R$ ). If we further adopt a $16 \%$ error for the foreground extinction (Schlegel et al. 1998), the formal combined error for a single $\bar{m}_{R}^{0}$ measurement is between 0.04 and $0.18 \mathrm{mag}$ (Col. 3). The error associated with the local colour (Col. 4) has been obtained through the usual error propagation formula from the uncertainties in the sky level determination, the photometry zero points, and Galactic extinction.

\section{SBF distances of the dE galaxies}

The results in Tables 6 and 7 show that for most galaxies the fluctuation magnitude $\bar{m}_{R}^{0}$ varies from field to field together with the local $(B-R)_{0}$ colour. This dependency was investigated by Jerjen et al. (2000, 2001) using Worthey's (1994) on-line model interpolation engine ${ }^{2}$ combined with the evolutionary isochrones from the Padova library (Bertelli et al. 1994). The

\footnotetext{
${ }^{2}$ http://199.120.161.183:80/ worthey/dial/ dial_a_pad.html
}

locus of theoretical $(B-R)_{0}$ and $\bar{m}_{R}^{0}$ values for mainly old stellar populations consists of two branches (Fig. 8). A parabolic branch stretching from $0.85<(B-R)_{0}<1.35$ is solely defined by single-burst, old $(>12 \mathrm{Gyr})$, metal-poor $([\mathrm{Fe} / \mathrm{H}]<-0.5)$ stellar populations. Slightly younger (8-12 Gyr), more metalrich populations or populations with a second burst of star formation fall onto a linear branch from $1.0<(B-R)_{0}<1.5$ (see also Fig. 8 of Jerjen et al. 2000). In their sample of Cen A group dwarfs, Jerjen et al. (2000) identified the dwarf ESO384-016 as "single-burst, old, metal-poor" type with data on the parabolic branch, whereas the three dEs ESO269-066, AM1339-445, and AM1343-452 produced data on the linear branch implying a younger and/or more complex underlying stellar population. These findings are consistent with the diversity of star formation histories revealed in Local Group (LG) dwarf ellipticals (Da Costa 1998; Grebel 1998). Moreover, the SBF data for Cen A group dwarfs provided first observational support for the predicted relative offset between the two theoretical branches.

An empirical zero point for the $(B-R)_{0}-\bar{m}_{R}^{0}$ relation was established from a comparison of SBF data for three M81 dwarf galaxies (Jerjen et al. 2001) with independent distances measured by Karachentsev and collaborators $(1999,2000)$ by means of the $I$-band magnitude of the red giant branch tip (TRGB). Lee et al. (1993) demonstrated that this method is a reliable distance indicator as relatively independent of age and metallicity. According to Da Costa \& Armandroff (1990), the TRGB is located at $M_{I}=-4.05 \pm 0.1 \mathrm{mag}$ for metal-poor 

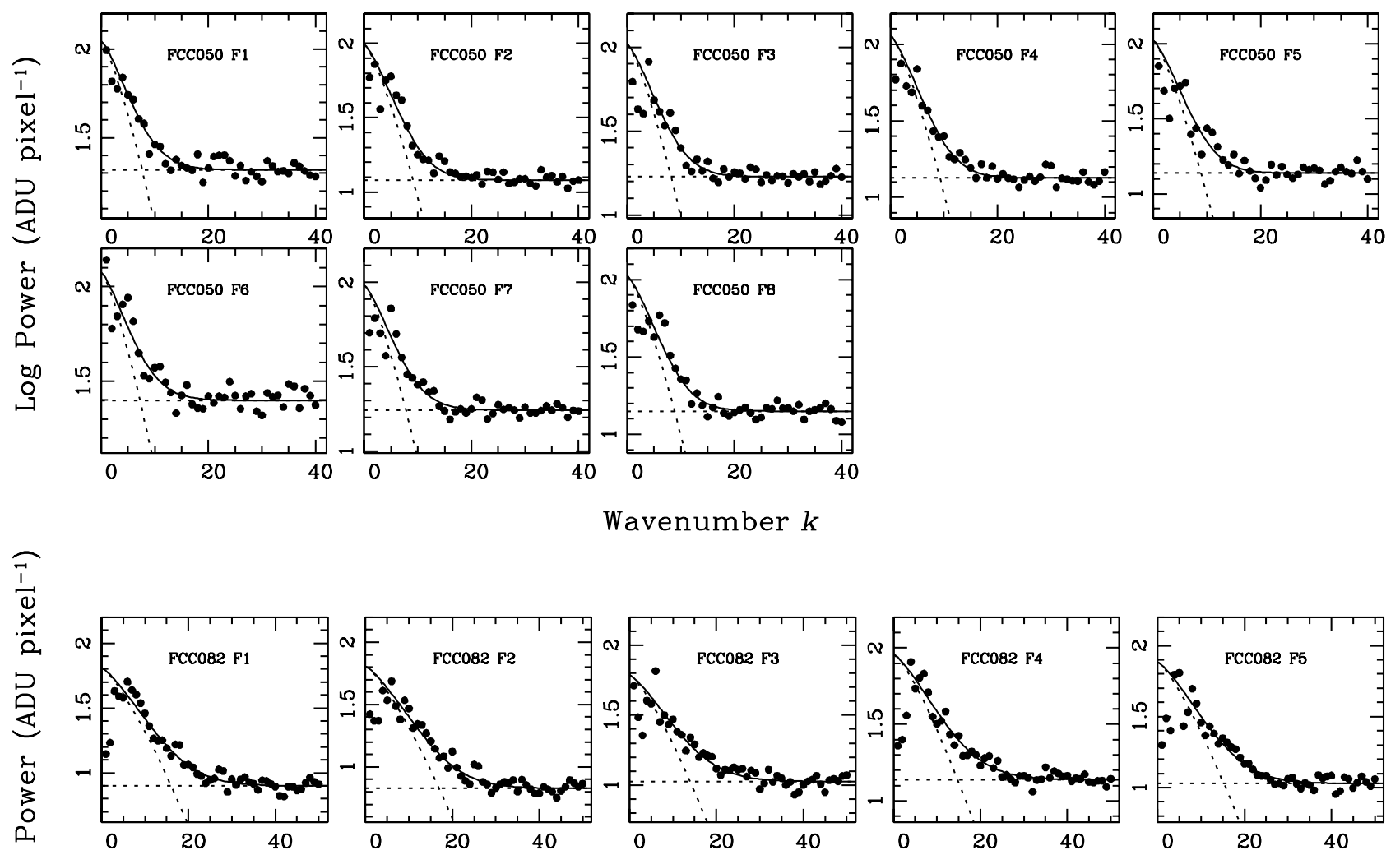

임

Wavenumber $k$
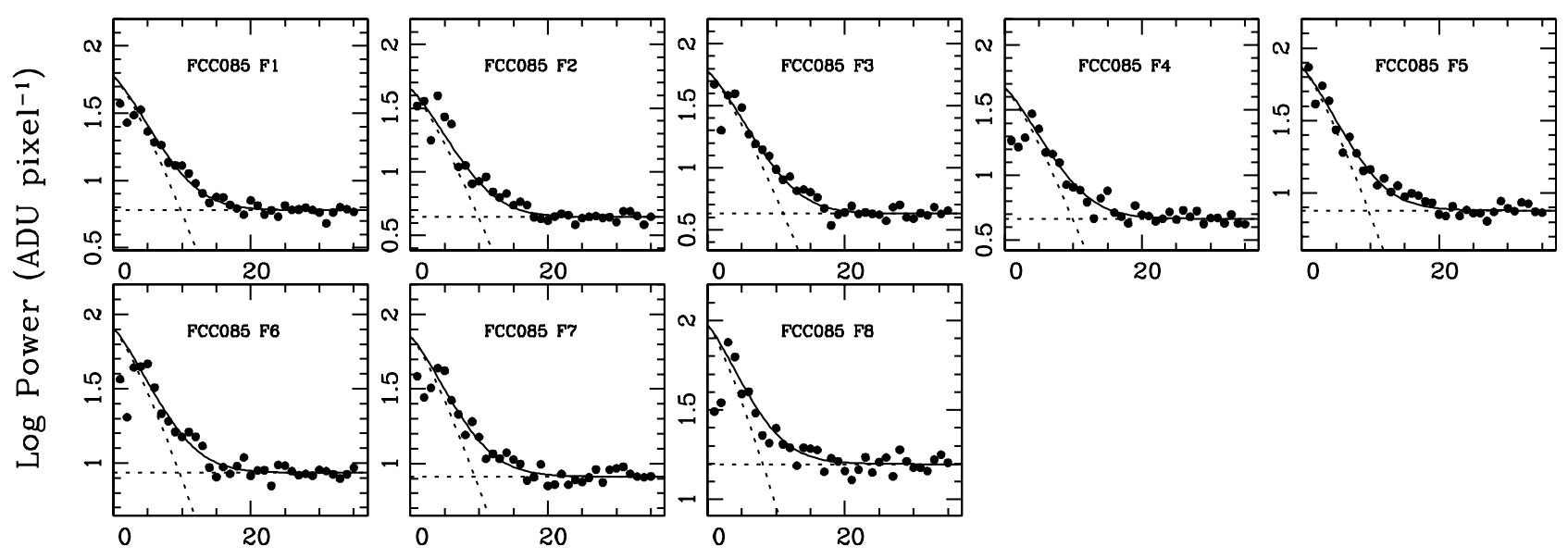

Wavenumber $k$

Fig. 4. The power spectra of all SBF fields analysed in the galaxies FCC050, FCC082, and FCC085.

systems, a calibration value that was recently confirmed by Ferrarese et al. (2000) from galaxies with Cepheid distances and by Bellazzini et al. (2001) based on photometry and a distance estimate from a detached eclipsing binary in the Galactic globular cluster Centauri.

The SBF and TRGB observations suggested a systematic underestimation of $\bar{M}_{R}$ by $0.13 \pm 0.03 \mathrm{mag}$. The origin of this discrepancy is not understood. But we refer the reader to Tonry et al. (2001) for a discussion of similar problems of the stellar evolution theory and population synthesis to reproduce the $I$-band SBF zero point. At the moment, the empirical $R$-band $\mathrm{SBF}$ calibration resting on the TRGB zero point appears the most reliable. Based on this value two analytical expressions were defined (Jerjen et al. 2001), one for each branch of the colour-fluctuation luminosity relation. They are currently used to calibrate $R$-band SBF data:

$$
\begin{array}{r}
\bar{M}_{R}=6.09(B-R)_{0}-8.94 \\
\quad \text { for } 1.00<(B-R)_{0}<1.35 \\
\bar{M}_{R}=1.89\left[(B-R)_{0}-0.77\right]^{2}-1.39 \\
\quad \text { for } 0.80<(B-R)_{0}<1.35
\end{array}
$$

Plotting $\bar{m}_{R}^{0}$ as a function of $(B-R)_{0}$ colour in Fig. 7 revealed a strong linear correlation between the two quantities for all 

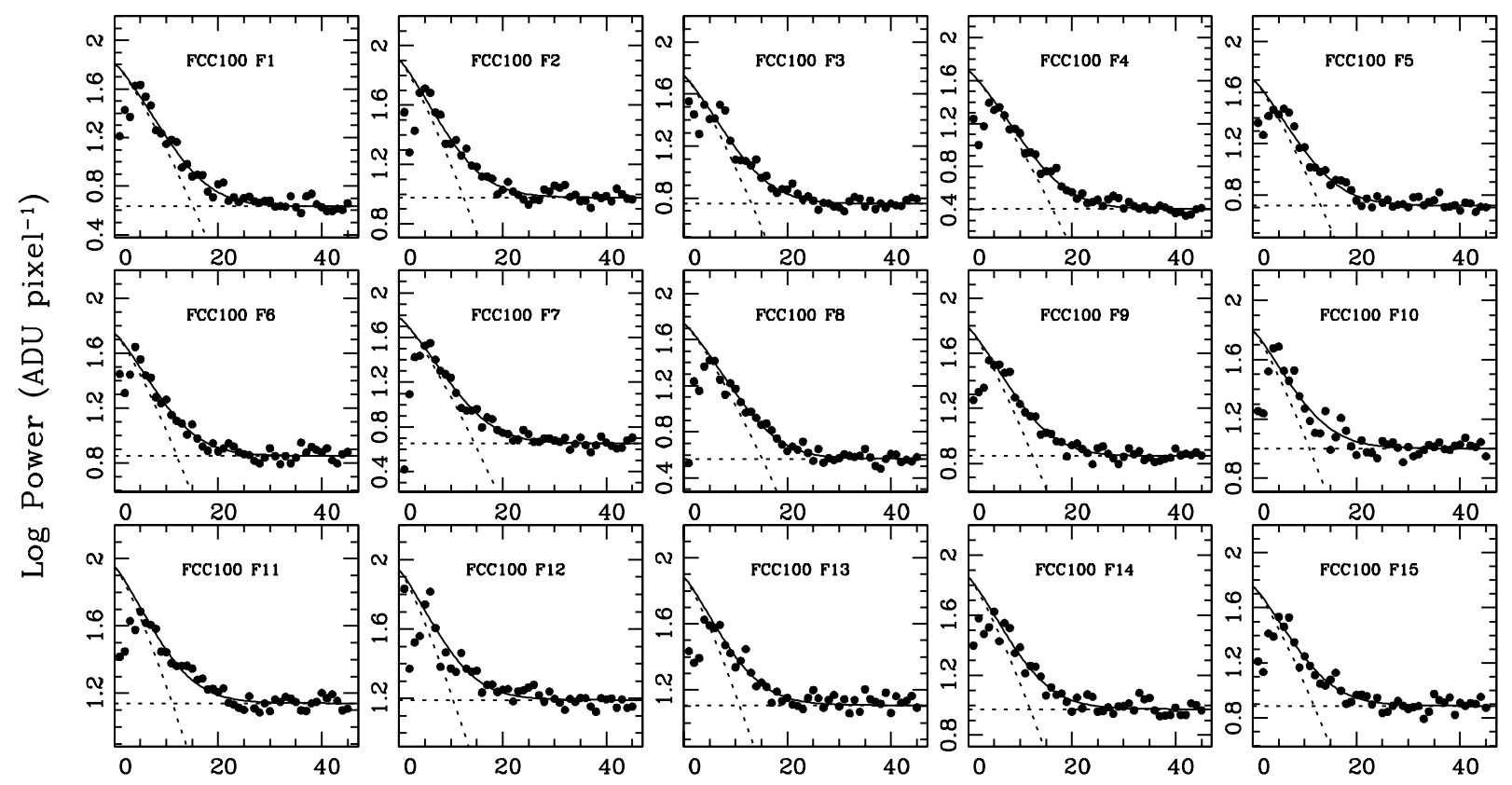

Wavenumber $k$
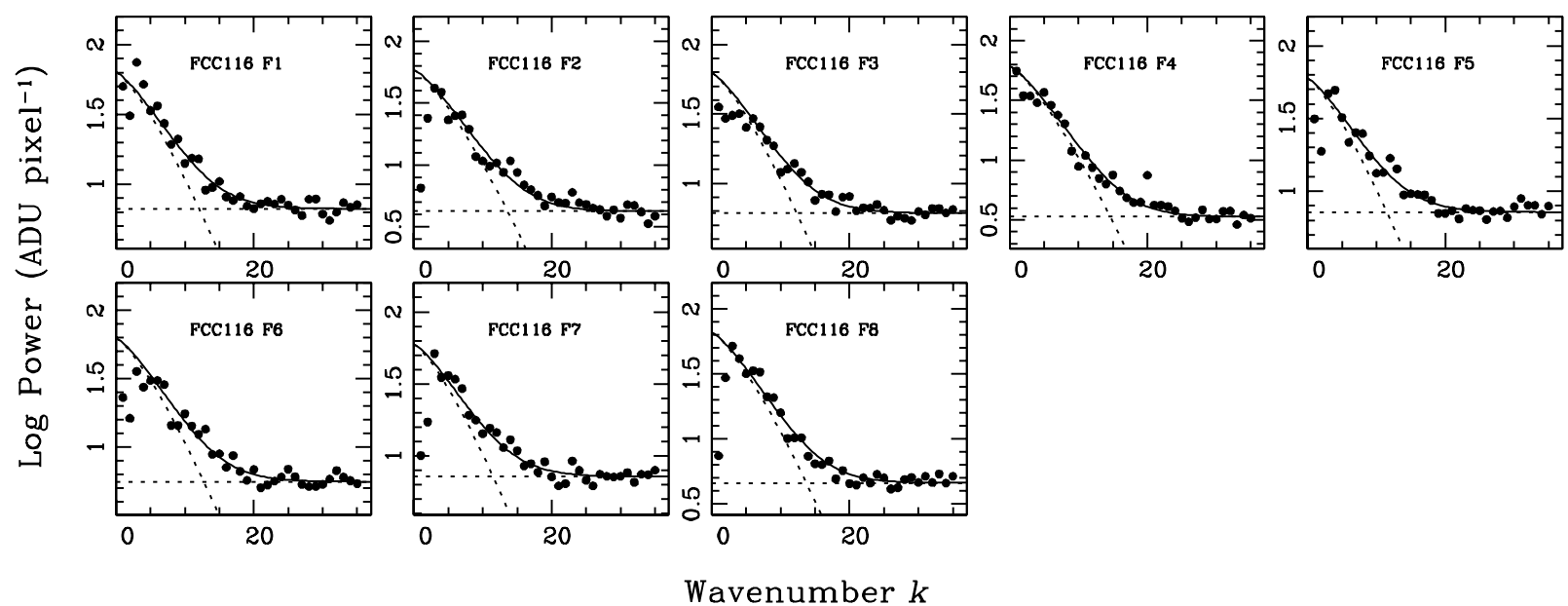

Fig. 5. The power spectra of all SBF fields analysed in the galaxies FCC100 and FC116.

galaxies but FCC116 which will be discussed separately. The linear trend demanded the use of Eq. (1) for the calibration of a data set by shifting the points simultaneously along the ordinate to find the best least squares fit. These best fits are shown in the panels of Fig. 7. The given error bars reflect the $1 \sigma$ uncertainty for a single measurement due to the sources discussed in Sect. 3. The corresponding distance moduli for the galaxies are compiled in Table 8 . The quoted overall distance errors there (Col. 3) also include the systematic error of $\pm 0.1 \mathrm{mag}$ from the TRGB zero point uncertainty.

FCC116 is a good example for the problem that occurs if no significant colour variation is detected among the analysed SBF fields in a galaxy. The lack of a clear trend in the $\left[(B-R)_{0}, \bar{m}_{R}^{0}\right]$ data makes it generally impossible to decide on which of the two branches to use for the calibration and thus prevents a secure distance measurement. However, we can utilize other arguments in the case of FCC116. The distance modulus of $30.95 \pm 0.19$ inferred from the parabolic branch is quite small moving this dwarf into the foreground of the Fornax cluster and $\sim 4.5 \mathrm{Mpc}$ away from its center. Such a short distance also appears incompatible with the observed velocity of $v_{\odot}=$ $1635 \mathrm{~km} \mathrm{~s}^{-1}$ for FCC116 that is only $150 \mathrm{~km} \mathrm{~s}^{-1}$ larger than the mean heliocentric velocity of the cluster (see below). Because of these reasons we feel confident that $(m-M)=31.30 \pm 0.19$ derived from the linear branch and formally consistent with the cluster center distance is the true distance modulus of FCC116.

\section{Distance and depth of the Fornax cluster}

Having SBF distances of eight genuine cluster galaxies, we can now turn to the estimate of the mean cluster distance. If the moduli of the dwarfs are weighted with their overall (random and systematic) errors (given in Col. 3 of Table 8) the derived mean distance is $(m-M)_{\text {Fornax }}=31.54 \mathrm{mag}$ (or $\left.D_{\text {Fornax }}=20.3 \mathrm{Mpc}\right)$. The dispersion of $\sigma_{(m-M)}=0.21 \mathrm{mag}$ yields a statistical uncertainty of $\pm 0.07 \mathrm{mag}$ ( or $\pm 0.7 \mathrm{Mpc}$ ). 

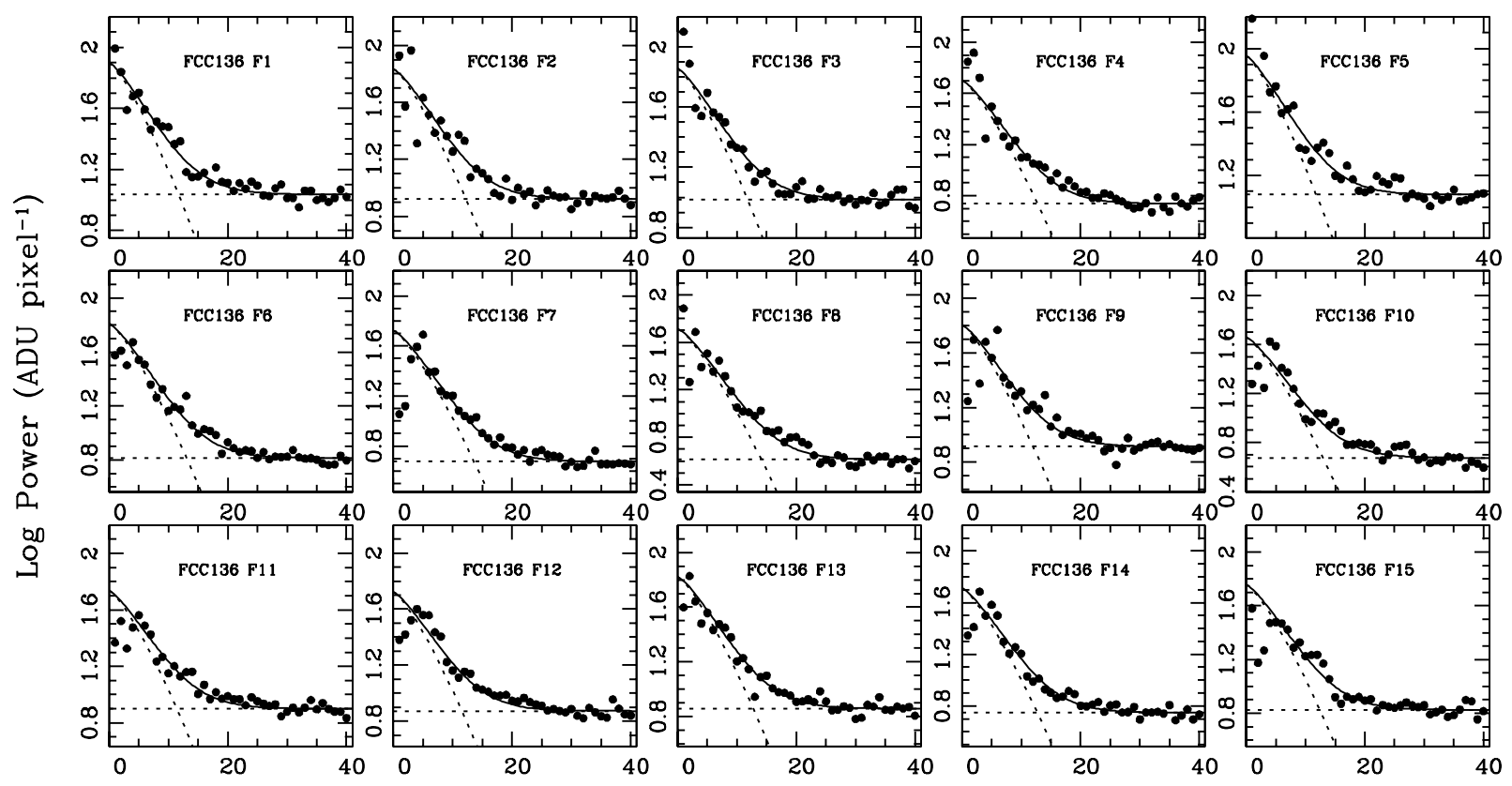

Wavenumber $k$
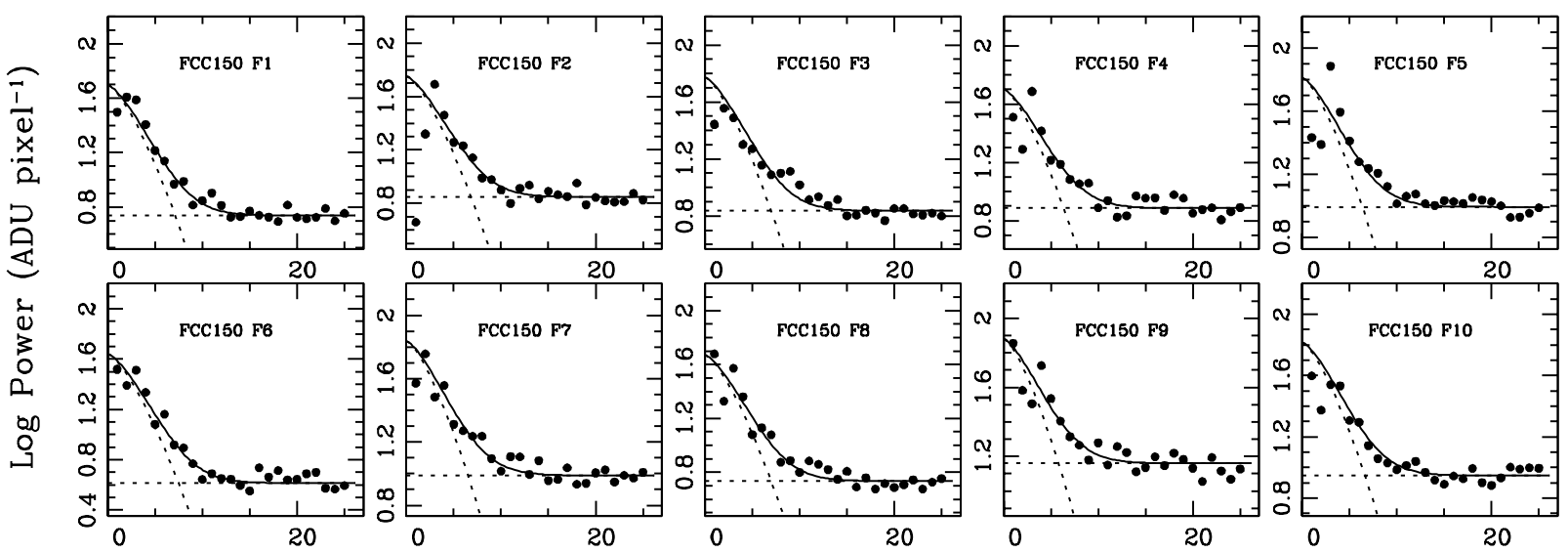

Wavenumber $k$

Fig. 6. The power spectra of all SBF fields analysed in the galaxies FFC136 and FCC150.

A comparison of this result with recent works on distances of other Fornax cluster galaxies reveals excellent agreement. An intercomparison of the $\mathrm{SBF}(\mathrm{R})$ distances for the $\mathrm{dE}$ galaxies with $\mathrm{SBF}(\mathrm{I})$ distances for 26 early-type giant galaxies in the Fornax region (Table 9, from Tonry et al. 2001) shows that both samples are statistically identical. The latter sample has a distance spread of $\sigma_{\mathrm{E} / \mathrm{S} 0}=0.21 \mathrm{mag}$ and a mean distance of $(m-M)_{\mathrm{E} / \mathrm{S} 0}=31.50 \pm 0.04 \mathrm{mag}$. For the one galaxy in common, FCC043 or IC1919, we find an agreement at the $1.3 \sigma$-level.

We like to emphasise that the 33 early-type galaxies compiled in the Tables 8 and 9 comprise a respectable fraction ( $\approx 10$ percent) of all cataloged Fornax cluster galaxies. As a consequence of the statistical similarities of the two data sets the mean distance modulus $(m-M)=31.51 \pm 0.04 \mathrm{mag}$ of the combined sample is insignificantly different from the individual results. Figure 9 visualises their distance distributions binned in $0.2 \mathrm{mag}$ intervals. The black area represents the dwarf ellipticals and the shaded area the giants. A strong concentration of galaxies at the mean modulus highlights the core region of the cluster.

At the bottom of Table 9 we also list the Cepheid distances published for the three Fornax cluster spirals NGC 1365 (Silbermann et al. 1999), NGC 1326A (Prosser et al. 1999), and NGC 1425 (Mould et al. 2000). Thereby the original distances were corrected for metallicity effects (Kelson et al. 2000). A great difficulty of using spiral galaxies to locate a cluster center is their trend to be preferentially found in the outskirts of clusters due to their late infall. Therefore, many more distances are required to compensate for the larger intrinsic distance spread of cluster spirals. The study of a carefully selected sample of spiral galaxies as done by the HST key project (Mould et al. 2000) certainly can help to reduce this problem but it never can be completely avoided as the quite different distance of NGC 1425 demonstrates. Mould et al. argued that instead of being a Fornax cluster member NGC 1425 could be 
Table 4. Parameters of the SBF analysis.

\begin{tabular}{|c|c|c|c|c|c|c|c|c|}
\hline $\begin{array}{l}\text { Name } \\
\text { (1) }\end{array}$ & $\begin{array}{c}\text { size } \\
\text { (pixels) } \\
(2)\end{array}$ & $\begin{array}{c}m_{1} \\
(\mathrm{mag}) \\
(3)\end{array}$ & $\begin{array}{c}\bar{g} \\
(\mathrm{ADU}) \\
(4)\end{array}$ & $\begin{array}{c}s \\
(\mathrm{ADU}) \\
(5)\end{array}$ & $\begin{array}{c}P_{0} \\
\left(\mathrm{ADU} \mathrm{s}^{-1} \mathrm{pixel}^{-1}\right) \\
(6)\end{array}$ & $\begin{array}{c}P_{1} \\
\left(\mathrm{ADU} \mathrm{s}^{-1} \text { pixel }^{-1}\right) \\
(7)\end{array}$ & $\begin{array}{l}S / N \\
(8)\end{array}$ & $\begin{array}{c}P_{\mathrm{BG}} / P_{0} \\
\text { (9) }\end{array}$ \\
\hline FCC043 F1 & 100 & 27.42 & 889.5 & 11816.0 & $0.063(0.001)$ & 0.009 & 5.1 & 0.04 \\
\hline$\ldots \mathrm{F} 2$ & & & 3651.1 & & $0.050(0.002)$ & 0.003 & 8.3 & 0.05 \\
\hline$\ldots \ldots \ldots \mathrm{F} 3$ & & & 1035.6 & & $0.060(0.002)$ & 0.008 & 5.3 & 0.05 \\
\hline$\ldots \ldots \ldots \mathrm{F} 4$ & & & 2701.0 & & $0.055(0.001)$ & 0.004 & 8.0 & 0.05 \\
\hline$\ldots \ldots \ldots \mathrm{F} 5$ & & & 593.1 & & $0.070(0.003)$ & 0.013 & 4.3 & 0.04 \\
\hline$\ldots \ldots \ldots \mathrm{F} 6$ & & & 3207.4 & & $0.054(0.002)$ & 0.003 & 8.6 & 0.05 \\
\hline$\ldots \ldots \ldots \mathrm{F} 7$ & & & 1103.7 & & $0.061(0.002)$ & 0.008 & 5.5 & 0.04 \\
\hline$\ldots \ldots \ldots \mathrm{F} 8$ & & & 644.0 & & $0.057(0.003)$ & 0.013 & 3.6 & 0.05 \\
\hline$\ldots \ldots \ldots \mathrm{F} 9$ & & & 550.6 & & $0.057(0.003)$ & 0.014 & 3.3 & 0.05 \\
\hline$\ldots \ldots$ F10 & & & 1209.1 & & $0.052(0.002)$ & 0.007 & 4.9 & 0.05 \\
\hline$\ldots \ldots . \mathrm{F} 11$ & & & 3855.5 & & $0.044(0.001)$ & 0.002 & 8.0 & 0.06 \\
\hline$\ldots \ldots$ F12 & & & 1371.9 & & $0.047(0.001)$ & 0.006 & 4.9 & 0.06 \\
\hline$\ldots \ldots . \mathrm{F} 13$ & & & 4143.1 & & $0.047(0.002)$ & 0.002 & 8.5 & 0.06 \\
\hline$\ldots \ldots \ldots$ F14 & & & 1417.5 & & $0.050(0.002)$ & 0.006 & 5.3 & 0.06 \\
\hline$\ldots \ldots$ F15 & & & 2974.4 & & $0.049(0.001)$ & 0.003 & 7.5 & 0.06 \\
\hline$\ldots \ldots$ F16 & & & 1524.4 & & $0.048(0.002)$ & 0.006 & 5.0 & 0.06 \\
\hline$\ldots \ldots \mathrm{F} 17$ & & & 2185.0 & & $0.052(0.002)$ & 0.005 & 6.8 & 0.05 \\
\hline$\ldots \ldots$ F18 & & & 1913.1 & & $0.049(0.002)$ & 0.005 & 5.9 & 0.06 \\
\hline .....F19 & & & 2091.4 & & $0.059(0.002)$ & 0.004 & 7.8 & 0.05 \\
\hline$\ldots \ldots$ F20 & & & 1254.9 & & $0.065(0.003)$ & 0.007 & 6.6 & 0.04 \\
\hline$\ldots \ldots . \mathrm{F} 21$ & & & 1079.7 & & $0.076(0.002)$ & 0.007 & 7.5 & 0.04 \\
\hline$\ldots \ldots$ F22 & & & 1475.7 & & $0.065(0.002)$ & 0.006 & 7.6 & 0.04 \\
\hline FCC050 F1 & 80 & 27.40 & 299.5 & 14291.0 & $0.158(0.011)$ & 0.030 & 4.8 & 0.02 \\
\hline$\ldots \ldots \ldots \mathrm{F} 2$ & & & 519.4 & & $0.141(0.012)$ & 0.017 & 7.0 & 0.02 \\
\hline$\ldots \ldots \ldots \mathrm{F} 3$ & & & 364.1 & & $0.150(0.012)$ & 0.024 & 5.5 & 0.02 \\
\hline$\ldots \ldots \ldots \mathrm{F} 4$ & & & 483.6 & & $0.163(0.014)$ & 0.019 & 7.4 & 0.02 \\
\hline$\ldots \ldots \ldots \mathrm{F} 5$ & & & 465.1 & & $0.150(0.022)$ & 0.020 & 6.6 & 0.02 \\
\hline$\ldots \ldots \ldots \mathrm{F} 6$ & & & 241.9 & & $0.170(0.018)$ & 0.036 & 4.3 & 0.02 \\
\hline$\ldots \ldots \ldots \mathrm{F} 7$ & & & 355.6 & & $0.138(0.016)$ & 0.025 & 4.9 & 0.02 \\
\hline$\ldots \ldots \ldots \mathrm{F} 8$ & & & 467.0 & & $0.151(0.015)$ & 0.020 & 6.5 & 0.02 \\
\hline FCC082 F1 & & 27.40 & 772.1 & 14248.0 & $0.092(0.003)$ & 0.011 & 6.4 & 0.03 \\
\hline$\ldots \ldots \ldots \mathrm{F} 2$ & & & 907.3 & & $0.091(0.004)$ & 0.010 & 7.3 & 0.03 \\
\hline$\ldots \ldots \ldots \mathrm{F} 3$ & & & 588.5 & & $0.087(0.004)$ & 0.015 & 4.8 & 0.03 \\
\hline$\ldots \ldots \ldots \mathrm{F} 4$ & & & 354.6 & & $0.129(0.005)$ & 0.020 & 5.7 & 0.02 \\
\hline$\ldots \ldots \ldots \mathrm{F} 5$ & & & 529.7 & & $0.109(0.006)$ & 0.015 & 6.0 & 0.02 \\
\hline FCC085 F1 & 70 & 27.40 & 1041.9 & 10581.0 & $0.085(0.002)$ & 0.009 & 7.3 & 0.03 \\
\hline$\ldots \ldots \ldots \mathrm{F} 2$ & & & 1301.5 & & $0.064(0.006)$ & 0.006 & 6.9 & 0.04 \\
\hline$\ldots \ldots \ldots \mathrm{F} 3$ & & & 1338.8 & & $0.085(0.004)$ & 0.006 & 9.5 & 0.03 \\
\hline$\ldots \ldots \ldots \mathrm{F} 4$ & & & 1119.0 & & $0.067(0.003)$ & 0.007 & 7.0 & 0.04 \\
\hline$\ldots \ldots \ldots \mathrm{F} 5$ & & & 681.5 & & $0.105(0.006)$ & 0.011 & 7.6 & 0.02 \\
\hline$\ldots \ldots \ldots \mathrm{F} 6$ & & & 572.1 & & $0.115(0.010)$ & 0.012 & 7.5 & 0.02 \\
\hline$\ldots \ldots \ldots \mathrm{F} 7$ & & & 695.4 & & $0.101(0.006)$ & 0.012 & 6.9 & 0.03 \\
\hline$\ldots \ldots \ldots \mathrm{F} 8$ & & & 299.9 & & $0.135(0.006)$ & 0.023 & 5.3 & 0.02 \\
\hline$\ldots \ldots \ldots \mathrm{F9}$ & & & 401.6 & & $0.118(0.005)$ & 0.018 & 5.5 & 0.02 \\
\hline
\end{tabular}

associated to the more distant Eridanus group for two reasons: (i) with coordinates $03 \mathrm{~h} 42 \mathrm{~m} 11.230 \mathrm{~s},-29 \mathrm{~d} 53 \mathrm{~m} 35.40 \mathrm{~s}$ (J2000), NGC 1425 is not found in the central region of Fornax but lies 5.6 degrees away from NGC 1399 towards Eridanus, and more importantly (ii) Fornax and Eridanus are overlapping in velocity space. If we exclude NGC 1425 on those grounds the remaining two Cepheid distances are consistent with the newly derived cluster distance.
It is now of interest to explore the possibility to estimate the line-of-sight depth of the Fornax cluster with our data. The observed dispersion $\sigma_{(m-M)}=0.21 \mathrm{mag}$ is influenced by two factors: (i) the intrinsic depth of the cluster along the line of sight $\left(\sigma_{\text {los }}\right)$ and (ii) the random distance error $\left(\sigma_{\Delta(m-M)}\right)$. The depth can thus be calculated with

$\sigma_{\text {los }}^{2}=\sigma_{(m-M)}^{2}-\sigma_{\Delta(m-M)}^{2}$, 
Table 5. Parameters of the SBF analysis.

\begin{tabular}{|c|c|c|c|c|c|c|c|c|}
\hline $\begin{array}{c}\text { Name } \\
(1)\end{array}$ & $\begin{array}{c}\text { size } \\
\text { (pixels) } \\
(2) \\
\end{array}$ & $\begin{array}{c}m_{1} \\
(\mathrm{mag}) \\
(3)\end{array}$ & $\begin{array}{c}\bar{g} \\
(\mathrm{ADU}) \\
(4) \\
\end{array}$ & $\begin{array}{c}s \\
(\mathrm{ADU}) \\
(5) \\
\end{array}$ & $\begin{array}{c}P_{0} \\
\left(\mathrm{ADU} \mathrm{s}^{-1} \text { pixel }^{-1}\right) \\
(6) \\
\end{array}$ & $\begin{array}{c}P_{1} \\
\left(\mathrm{ADU} \mathrm{s}^{-1} \mathrm{pixel}^{-1}\right) \\
(7) \\
\end{array}$ & $\begin{array}{l}S / N \\
(8) \\
\end{array}$ & $\begin{array}{c}P_{\mathrm{BG}} / P_{0} \\
(9) \\
\end{array}$ \\
\hline FCC100 F1 & 100 & 27.41 & 1133.9 & 9044.0 & $0.092(0.004)$ & 0.006 & 10.2 & 0.03 \\
\hline$\ldots \ldots \ldots \mathrm{F} 2$ & & & 427.8 & & $0.115(0.005)$ & 0.014 & 6.9 & 0.02 \\
\hline$\ldots \ldots \ldots \mathrm{F} 3$ & & & 784.3 & & $0.079(0.004)$ & 0.008 & 7.1 & 0.03 \\
\hline$\ldots \ldots \ldots \mathrm{F} 4$ & & & 2000.2 & & $0.070(0.003)$ & 0.004 & 10.7 & 0.04 \\
\hline$\ldots \ldots \ldots \mathrm{F} 5$ & & & 839.3 & & $0.072(0.003)$ & 0.007 & 6.9 & 0.04 \\
\hline .......F6 & & & 630.0 & & $0.079(0.003)$ & 0.010 & 6.0 & 0.03 \\
\hline$\ldots \ldots \ldots \mathrm{F} 7$ & & & 1245.0 & & $0.086(0.004)$ & 0.006 & 9.1 & 0.03 \\
\hline$\ldots \ldots \ldots \mathrm{F} 8$ & & & 1474.8 & & $0.078(0.003)$ & 0.005 & 9.6 & 0.03 \\
\hline$\ldots \ldots \ldots \mathrm{F} 9$ & & & 598.4 & & $0.087(0.004)$ & 0.010 & 6.6 & 0.03 \\
\hline$\ldots \ldots \ldots \mathrm{F} 10$ & & & 385.2 & & $0.089(0.005)$ & 0.014 & 5.1 & 0.03 \\
\hline$\ldots \ldots$ F11 & & & 283.5 & & $0.128(0.006)$ & 0.020 & 5.6 & 0.02 \\
\hline$\ldots \ldots \ldots \mathrm{F} 12$ & & & 263.9 & & $0.124(0.013)$ & 0.022 & 4.9 & 0.02 \\
\hline$\ldots \ldots \ldots \mathrm{F} 13$ & & & 342.4 & & $0.109(0.007)$ & 0.018 & 5.1 & 0.02 \\
\hline$\ldots \ldots$ F14 & & & 462.8 & & $0.102(0.005)$ & 0.013 & 6.1 & 0.03 \\
\hline$\ldots \ldots$ F15 & & & 593.7 & & $0.081(0.004)$ & 0.011 & 5.7 & 0.03 \\
\hline FCC116 F1 & 70 & 27.39 & 1074.3 & 12684.0 & $0.090(0.003)$ & 0.010 & 7.3 & 0.03 \\
\hline$\ldots \ldots \ldots \mathrm{F} 2$ & & & 1674.1 & & $0.084(0.004)$ & 0.006 & 9.4 & 0.03 \\
\hline$\ldots \ldots \ldots \mathrm{F} 3$ & & & 979.0 & & $0.089(0.003)$ & 0.009 & 7.6 & 0.03 \\
\hline$\ldots \ldots \ldots \mathrm{F} 4$ & & & 1896.3 & & $0.087(0.004)$ & 0.005 & 11.4 & 0.03 \\
\hline ......F5 & & & 901.2 & & $0.085(0.004)$ & 0.010 & 6.4 & 0.03 \\
\hline ......F6 & & & 1237.9 & & $0.089(0.005)$ & 0.008 & 8.2 & 0.03 \\
\hline$\ldots \ldots \ldots \mathrm{F} 7$ & & & 879.7 & & $0.085(0.004)$ & 0.010 & 6.4 & 0.03 \\
\hline$\ldots \ldots \ldots \mathrm{F} 8$ & & & 1559.6 & & $0.094(0.004)$ & 0.007 & 10.0 & 0.03 \\
\hline FCC136 F1 & 80 & 27.38 & 415.2 & 11376.0 & $0.114(0.007)$ & 0.016 & 6.2 & 0.02 \\
\hline$\ldots \ldots \ldots \mathrm{F} 2$ & & & 567.9 & & $0.098(0.007)$ & 0.012 & 6.6 & 0.03 \\
\hline$\ldots \ldots \ldots \mathrm{F} 3$ & & & 546.3 & & $0.103(0.006)$ & 0.014 & 6.1 & 0.02 \\
\hline$\ldots \ldots \ldots \mathrm{F} 4$ & & & 1067.2 & & $0.072(0.004)$ & 0.008 & 6.7 & 0.04 \\
\hline$\ldots \ldots \ldots \mathrm{F} 5$ & & & 419.7 & & $0.129(0.010)$ & 0.017 & 6.4 & 0.02 \\
\hline ......F6 & & & 807.1 & & $0.092(0.005)$ & 0.009 & 7.6 & 0.03 \\
\hline$\ldots \ldots \ldots \mathrm{F} 7$ & & & 1350.2 & & $0.076(0.012)$ & 0.007 & 7.9 & 0.03 \\
\hline$\ldots \ldots \ldots \mathrm{F} 8$ & & & 1622.8 & & $0.074(0.006)$ & 0.006 & 8.5 & 0.03 \\
\hline$\ldots \ldots \ldots \mathrm{F} 9$ & & & 765.5 & & $0.092(0.005)$ & 0.012 & 6.2 & 0.03 \\
\hline .....F10 & & & 1265.4 & & $0.065(0.006)$ & 0.007 & 6.7 & 0.04 \\
\hline$\ldots \ldots \ldots 11$ & & & 731.1 & & $0.078(0.005)$ & 0.011 & 5.4 & 0.03 \\
\hline$\ldots \ldots \ldots \mathrm{F} 12$ & & & 796.2 & & $0.075(0.004)$ & 0.011 & 5.5 & 0.03 \\
\hline$\ldots \ldots \ldots \mathrm{F} 13$ & & & 785.6 & & $0.095(0.004)$ & 0.010 & 7.2 & 0.03 \\
\hline$\ldots \ldots \ldots$ F14 & & & 1007.2 & & $0.074(0.004)$ & 0.008 & 6.7 & 0.03 \\
\hline$\ldots \ldots \ldots$ F15 & & & 842.7 & & $0.082(0.004)$ & 0.010 & 6.5 & 0.03 \\
\hline FCC150 F1 & 50 & 27.27 & 4754.6 & 16598.0 & $0.071(0.007)$ & 0.008 & 7.0 & 0.03 \\
\hline$\ldots \ldots \ldots \mathrm{F} 2$ & & & 3743.5 & & $0.081(0.009)$ & 0.010 & 6.5 & 0.03 \\
\hline$\ldots \ldots \ldots \mathrm{F} 3$ & & & 3451.4 & & $0.085(0.011)$ & 0.010 & 7.0 & 0.02 \\
\hline$\ldots \ldots \ldots \mathrm{F} 4$ & & & 3058.3 & & $0.072(0.008)$ & 0.011 & 5.4 & 0.03 \\
\hline$\ldots \ldots \ldots \mathrm{F} 5$ & & & 2162.2 & & $0.093(0.011)$ & 0.014 & 5.6 & 0.02 \\
\hline ......F6 & & & 5870.8 & & $0.063(0.006)$ & 0.006 & 7.6 & 0.03 \\
\hline$\ldots \ldots \ldots \mathrm{F} 7$ & & & 1759.4 & & $0.098(0.017)$ & 0.014 & 6.0 & 0.02 \\
\hline$\ldots \ldots \ldots \mathrm{F} 8$ & & & 4302.8 & & $0.067(0.006)$ & 0.008 & 6.6 & 0.03 \\
\hline$\ldots \ldots \ldots \mathrm{F} 9$ & & & 1853.8 & & $0.109(0.011)$ & 0.021 & 4.7 & 0.02 \\
\hline$\ldots \ldots \ldots$ F10 & & & 2084.2 & & $0.094(0.006)$ & 0.013 & 6.2 & 0.02 \\
\hline
\end{tabular}

provided that $\sigma_{\Delta(m-M)}$ represents a robust estimate of the mean distance error of our sample. As the latter is not well defined due to some variation in distance accuracy we generated 100000 bootstrap samples (Efron \& Tibshirani 1986) of size eight drawn from the original data. For each sample we calculated the corresponding three quantities, $\sigma_{(m-M)}^{2}$, the square of the median error $\sigma_{\Delta(m-M)}^{2}$, and $\sigma_{\text {los }}^{2}$. It should be stressed that the search for $\sigma_{\text {los }}$ requires only relative distances. 

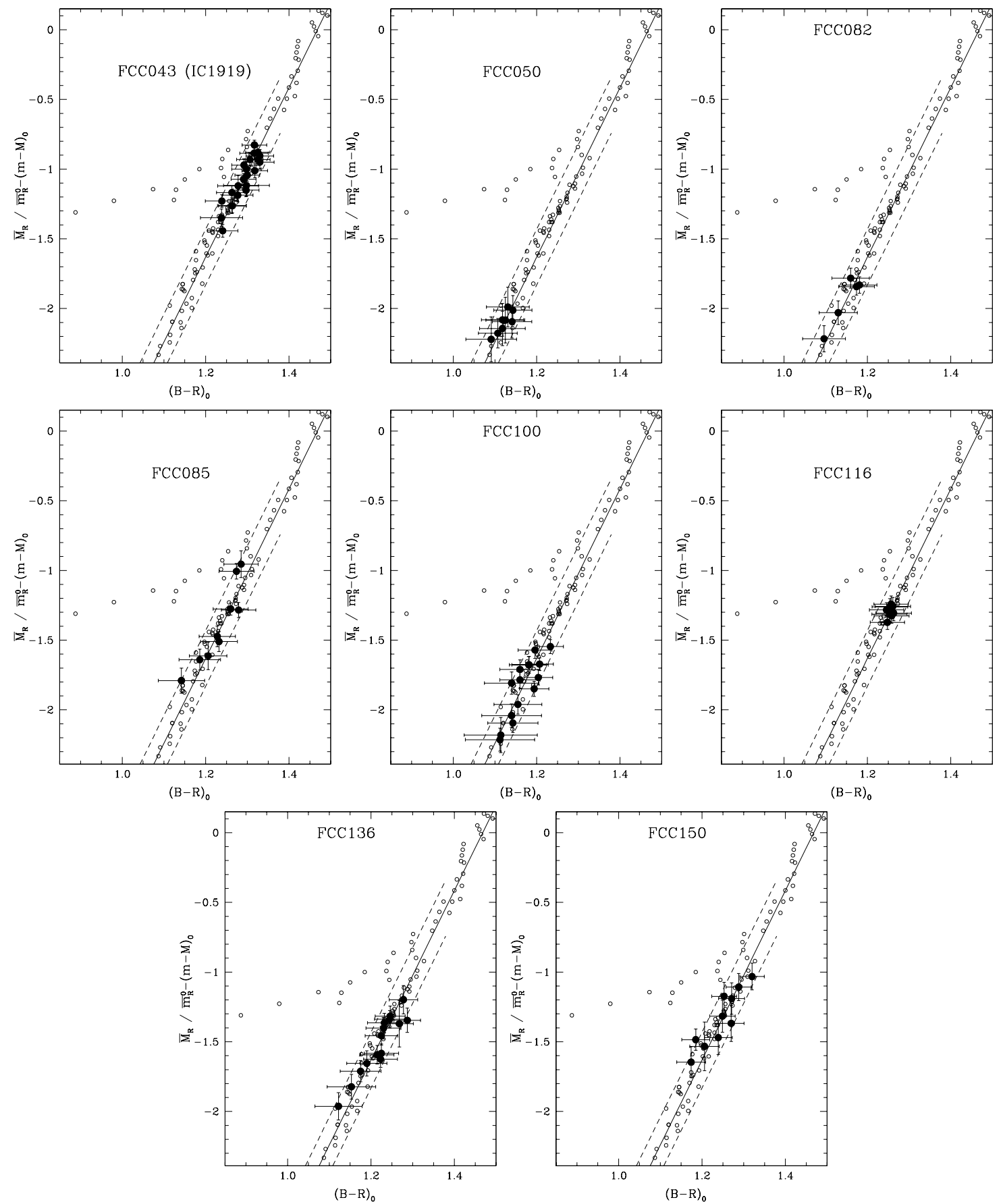

Fig. 7. The $\left[(B-R)_{0}, \bar{m}_{R}^{0}\right]$ data for each galaxy was shifted to the locus of the theoretical $(B-R)-\bar{M}_{R}$ relation to measure the distance modulus. The observed correlations between the fluctuation magnitudes and the local $(B-R)$ colours follow closely the linear branch of the model locus. The dashed lines above and below the branch indicate the \pm 0.2 mag strip that envelopes the intrinsic scatter from different models and the observed scatter in the fluctuation measurements.

Therefore, we could use the smaller random distance errors (Col. 4, Table 8) instead of the overall errors that also include the uncertainty of the TRGB zero point. The distribution of $\sigma_{\text {los }}^{2}$ is well approximated by a Gaussian with a peak at 0.0209 and a $1 \sigma$ scatter of 0.0165 . These numbers translate into a $1.8 \sigma$ detection of the line-of-sight cluster depth $\sigma_{\text {los }}=1.4_{-0.8}^{+0.5} \mathrm{Mpc}$.

Considering the small number of galaxies involved in the analysis the result is still provided with a large uncertainty. Nevertheless, we can draw two conclusions. Firstly, the depth seems to be in agreement with the compact appearance of the Fornax cluster in the sky. The positions of the early-type galaxies taken from the FCC show a dispersion of $\sigma \approx 0.5 \mathrm{Mpc}$ in the two other dimensions (RA and DEC). Secondly, there is the perspective of analysing a larger sample of $25 \mathrm{dEs}$ (see Sect. 1) in the near future. This will allow to reduce the current uncertainty of $\sigma_{\text {los }}$ by $\sim 40-50 \%$ which will put the final result on firmer grounds. 
Table 6. Fluctuation magnitudes and local colours for each SBF field in FCC043, FCC050, FCC082, and FCC085 after correction for Galactic extinction.

\begin{tabular}{|c|c|c|c|}
\hline $\begin{array}{c}\text { Name } \\
\text { (1) }\end{array}$ & $\begin{array}{c}A_{R} \\
(\mathrm{mag}) \\
(2)\end{array}$ & $\begin{array}{c}\bar{m}_{R}^{0} \\
(\mathrm{mag}) \\
(3)\end{array}$ & $\begin{array}{c}(B-R)_{0} \\
(\mathrm{mag}) \\
\quad(4)\end{array}$ \\
\hline FCC043 F1 & $0.04 \pm 0.01$ & $30.43 \pm 0.05$ & $1.24 \pm 0.03$ \\
\hline$\ldots \ldots \ldots \mathrm{F} 2$ & & $30.69 \pm 0.04$ & $1.29 \pm 0.01$ \\
\hline$\ldots \ldots \ldots \mathrm{F} 3$ & & $30.49 \pm 0.05$ & $1.26 \pm 0.02$ \\
\hline$\ldots \ldots \mathrm{F} 4$ & & $30.59 \pm 0.04$ & $1.29 \pm 0.01$ \\
\hline ......F5 & & $30.31 \pm 0.06$ & $1.24 \pm 0.04$ \\
\hline ......F6 & & $30.62 \pm 0.04$ & $1.30 \pm 0.01$ \\
\hline$\ldots \ldots \ldots \mathrm{F} 7$ & & $30.47 \pm 0.05$ & $1.28 \pm 0.02$ \\
\hline$\ldots \ldots \ldots \mathrm{F} 8$ & & $30.54 \pm 0.07$ & $1.28 \pm 0.04$ \\
\hline$\ldots \ldots \ldots \mathrm{F} 9$ & & $30.54 \pm 0.08$ & $1.30 \pm 0.05$ \\
\hline ...FF10 & & $30.66 \pm 0.05$ & $1.30 \pm 0.02$ \\
\hline .....FF11 & & $30.83 \pm 0.04$ & $1.32 \pm 0.01$ \\
\hline .....FF12 & & $30.77 \pm 0.05$ & $1.32 \pm 0.02$ \\
\hline ......F13 & & $30.78 \pm 0.05$ & $1.32 \pm 0.01$ \\
\hline .....FF14 & & $30.71 \pm 0.05$ & $1.33 \pm 0.02$ \\
\hline .....FF15 & & $30.73 \pm 0.04$ & $1.32 \pm 0.01$ \\
\hline ...F16 & & $30.76 \pm 0.05$ & $1.33 \pm 0.02$ \\
\hline .....F17 & & $30.65 \pm 0.05$ & $1.32 \pm 0.01$ \\
\hline ......F18 & & $30.73 \pm 0.05$ & $1.31 \pm 0.01$ \\
\hline .....FF19 & & $30.51 \pm 0.05$ & $1.30 \pm 0.01$ \\
\hline ......F20 & & $30.40 \pm 0.06$ & $1.26 \pm 0.02$ \\
\hline$\ldots \ldots \ldots \mathrm{F} 21$ & & $30.22 \pm 0.05$ & $1.24 \pm 0.02$ \\
\hline ......F22 & & $30.40 \pm 0.05$ & $1.26 \pm 0.02$ \\
\hline FCC050 F1 & $0.03 \pm 0.01$ & $29.40 \pm 0.12$ & $1.12 \pm 0.05$ \\
\hline$\ldots \ldots \ldots \mathrm{F} 2$ & & $29.53 \pm 0.10$ & $1.14 \pm 0.04$ \\
\hline$\ldots \ldots \ldots \mathrm{F} 3$ & & $29.46 \pm 0.12$ & $1.12 \pm 0.04$ \\
\hline$\ldots \ldots \ldots \mathrm{F} 4$ & & $29.36 \pm 0.10$ & $1.11 \pm 0.04$ \\
\hline$\ldots$ F5 & & $29.46 \pm 0.16$ & $1.13 \pm 0.04$ \\
\hline ....F6 & & $29.32 \pm 0.16$ & $1.09 \pm 0.05$ \\
\hline$\ldots \ldots \ldots \mathrm{F} 7$ & & $29.55 \pm 0.14$ & $1.13 \pm 0.04$ \\
\hline$\ldots \ldots \ldots \mathrm{F} 8$ & & $29.45 \pm 0.12$ & $1.14 \pm 0.04$ \\
\hline FCC082 F1 & $0.04 \pm 0.01$ & $29.98 \pm 0.06$ & $1.17 \pm 0.03$ \\
\hline$\ldots \ldots \ldots \mathrm{F} 2$ & & $29.99 \pm 0.06$ & $1.18 \pm 0.03$ \\
\hline$\ldots \ldots \ldots \mathrm{F} 3$ & & $30.04 \pm 0.07$ & $1.16 \pm 0.03$ \\
\hline$\ldots \ldots \ldots \mathrm{F} 4$ & & $29.60 \pm 0.09$ & $1.10 \pm 0.04$ \\
\hline ......F5 & & $29.79 \pm 0.08$ & $1.13 \pm 0.04$ \\
\hline FCC085 F1 & $0.04 \pm 0.01$ & $30.07 \pm 0.04$ & $1.27 \pm 0.03$ \\
\hline$\ldots \ldots \ldots \mathrm{F} 2$ & & $30.39 \pm 0.10$ & $1.29 \pm 0.03$ \\
\hline$\ldots . . F 3$ & & $30.07 \pm 0.05$ & $1.28 \pm 0.03$ \\
\hline$\ldots \ldots \ldots \mathrm{F} 4$ & & $30.34 \pm 0.06$ & $1.28 \pm 0.03$ \\
\hline$\ldots \ldots \ldots \mathrm{F} 5$ & & $29.84 \pm 0.07$ & $1.24 \pm 0.03$ \\
\hline ......F6 & & $29.74 \pm 0.10$ & $1.22 \pm 0.04$ \\
\hline$\ldots \ldots \ldots \mathrm{F} 7$ & & $29.88 \pm 0.07$ & $1.24 \pm 0.03$ \\
\hline$\ldots \ldots \ldots \mathrm{F} 8$ & & $29.56 \pm 0.09$ & $1.16 \pm 0.05$ \\
\hline ......F9 & & $29.71 \pm 0.07$ & $1.20 \pm 0.04$ \\
\hline
\end{tabular}

\section{The Hubble constant at the Fornax cluster}

We found in the previous section a statistically robust distance to the Fornax cluster that was based on dwarf elliptical galaxies. This value shall now be used to estimate the Hubble constant $H_{0}$. A reliable method is to simply divide the cosmo-
Table 7. Fluctuation magnitudes and local colours for each SBF field in FCC100, FCC116, FCC136, and FCC150 after correction for Galactic extinction.

\begin{tabular}{|c|c|c|c|}
\hline $\begin{array}{c}\text { Name } \\
\text { (1) }\end{array}$ & $\begin{array}{c}A_{R} \\
(\mathrm{mag}) \\
(2)\end{array}$ & $\begin{array}{c}\bar{m}_{R}^{0} \\
(\mathrm{mag}) \\
(3)\end{array}$ & $\begin{array}{c}(B-R)_{0} \\
(\mathrm{mag}) \\
(4)\end{array}$ \\
\hline FCC100 F1 & $0.04 \pm 0.01$ & $29.99 \pm 0.05$ & $1.19 \pm 0.02$ \\
\hline ..F2 & & $29.75 \pm 0.07$ & $1.14 \pm 0.05$ \\
\hline$\ldots \ldots \mathrm{F} 3$ & & $30.16 \pm 0.06$ & $1.18 \pm 0.03$ \\
\hline$\ldots \ldots \ldots \mathrm{F} 4$ & & $30.29 \pm 0.05$ & $1.23 \pm 0.01$ \\
\hline$\ldots \ldots \ldots \mathrm{F} 5$ & & $30.27 \pm 0.06$ & $1.20 \pm 0.03$ \\
\hline ....F6 & & $30.16 \pm 0.06$ & $1.18 \pm 0.04$ \\
\hline$\ldots \ldots \ldots \mathrm{F} 7$ & & $30.07 \pm 0.05$ & $1.20 \pm 0.02$ \\
\hline$\ldots \ldots \ldots \mathrm{F} 8$ & & $30.17 \pm 0.04$ & $1.21 \pm 0.02$ \\
\hline$\ldots$...F9 & & $30.05 \pm 0.06$ & $1.16 \pm 0.04$ \\
\hline .....F10 & & $30.03 \pm 0.08$ & $1.14 \pm 0.06$ \\
\hline .....FF11 & & $29.63 \pm 0.08$ & $1.11 \pm 0.08$ \\
\hline .....F12 & & $29.66 \pm 0.13$ & $1.11 \pm 0.08$ \\
\hline .....F13 & & $29.80 \pm 0.08$ & $1.14 \pm 0.07$ \\
\hline .....F14 & & $29.88 \pm 0.07$ & $1.15 \pm 0.05$ \\
\hline ..F15 & & $30.13 \pm 0.07$ & $1.16 \pm 0.04$ \\
\hline FCC116 F1 & $0.06 \pm 0.01$ & $29.97 \pm 0.05$ & $1.26 \pm 0.03$ \\
\hline$\ldots \ldots \ldots \mathrm{F} 2$ & & $30.06 \pm 0.06$ & $1.26 \pm 0.03$ \\
\hline$\ldots \mathrm{F} 3$ & & $29.99 \pm 0.05$ & $1.26 \pm 0.03$ \\
\hline$\ldots \ldots \ldots \mathrm{F} 4$ & & $30.02 \pm 0.05$ & $1.25 \pm 0.03$ \\
\hline$\ldots \ldots$ F5 & & $30.04 \pm 0.06$ & $1.25 \pm 0.03$ \\
\hline .......F6 & & $29.99 \pm 0.06$ & $1.25 \pm 0.03$ \\
\hline$\ldots \ldots \ldots \mathrm{F} 7$ & & $30.04 \pm 0.06$ & $1.26 \pm 0.03$ \\
\hline$\ldots \ldots \ldots \mathrm{F} 8$ & & $29.93 \pm 0.05$ & $1.25 \pm 0.03$ \\
\hline FCC136 F1 & $0.04 \pm 0.01$ & $29.73 \pm 0.09$ & $1.15 \pm 0.05$ \\
\hline$\ldots \mathrm{F} 2$ & & $29.89 \pm 0.09$ & $1.19 \pm 0.04$ \\
\hline$\ldots \ldots \ldots \mathrm{F} 3$ & & $29.84 \pm 0.08$ & $1.17 \pm 0.04$ \\
\hline$\ldots \ldots \ldots \mathrm{F} 4$ & & $30.23 \pm 0.07$ & $1.25 \pm 0.02$ \\
\hline$\ldots \mathrm{F} 5$ & & $29.59 \pm 0.10$ & $1.12 \pm 0.05$ \\
\hline .......F6 & & $29.96 \pm 0.07$ & $1.22 \pm 0.03$ \\
\hline$\ldots$ F7 & & $30.18 \pm 0.17$ & $1.27 \pm 0.02$ \\
\hline$\ldots \mathrm{F} 8$ & & $30.20 \pm 0.09$ & $1.29 \pm 0.02$ \\
\hline$\ldots \ldots \ldots \mathrm{F} 9$ & & $29.97 \pm 0.07$ & $1.23 \pm 0.03$ \\
\hline .....F10 & & $30.35 \pm 0.10$ & $1.28 \pm 0.02$ \\
\hline$\ldots F 11$ & & $30.15 \pm 0.08$ & $1.23 \pm 0.03$ \\
\hline .....F 12 & & $30.19 \pm 0.07$ & $1.23 \pm 0.03$ \\
\hline$\ldots F 13$ & & $29.92 \pm 0.06$ & $1.22 \pm 0.03$ \\
\hline ..F14 & & $30.21 \pm 0.06$ & $1.24 \pm 0.02$ \\
\hline .....F15 & & $30.09 \pm 0.07$ & $1.22 \pm 0.03$ \\
\hline FCC150 F1 & $0.04 \pm 0.01$ & $30.14 \pm 0.10$ & $1.25 \pm 0.01$ \\
\hline$\ldots \ldots \ldots \mathrm{F} 2$ & & $29.99 \pm 0.12$ & $1.25 \pm 0.01$ \\
\hline$\ldots \ldots \ldots \mathrm{F} 3$ & & $29.94 \pm 0.13$ & $1.27 \pm 0.01$ \\
\hline$\ldots \ldots \ldots \mathrm{F} 4$ & & $30.12 \pm 0.11$ & $1.27 \pm 0.01$ \\
\hline$\ldots$. F5 & & $29.84 \pm 0.12$ & $1.24 \pm 0.02$ \\
\hline$\ldots \mathrm{F} 6$ & & $30.28 \pm 0.10$ & $1.32 \pm 0.01$ \\
\hline$\ldots \ldots \ldots \mathrm{F} 7$ & & $29.78 \pm 0.18$ & $1.21 \pm 0.02$ \\
\hline$\ldots \mathrm{F} 8$ & & $30.20 \pm 0.10$ & $1.29 \pm 0.01$ \\
\hline .......F9 & & $29.66 \pm 0.11$ & $1.17 \pm 0.02$ \\
\hline .....F10 & & $29.83 \pm 0.08$ & $1.18 \pm 0.02$ \\
\hline
\end{tabular}

logical velocity of Fornax $v_{\mathrm{CMB}}^{\text {Fornax }}$ by the cluster distance. The former quantity can be derived from a recent measurement of the mean heliocentric velocity of Fornax by Drinkwater et al. (2001). These authors reported $v_{\odot}^{\text {Fornax }}=1493 \pm$ $36 \mathrm{~km} \mathrm{~s}^{-1}$ based on 108 galaxy redshifts. The observed velocity 


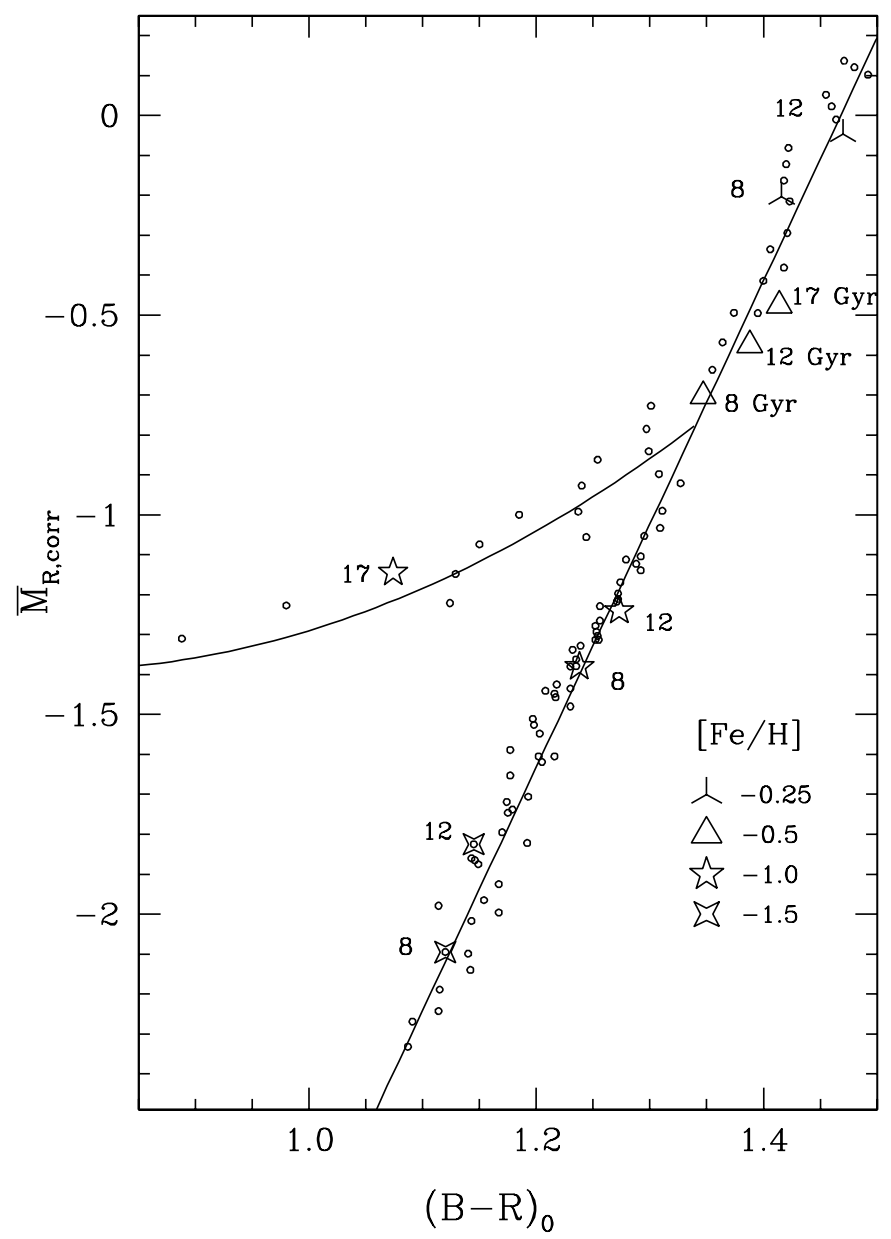

Fig. 8. Worthey+Padova model predictions of a $(B-R)_{0}$ colour- $R$-band fluctuation luminosity relation for a grid of stellar populations (open circles and symbols): a set of single burst populations that covers the $\{$ age $=8,12,17 \mathrm{Gyr}\} \times\{[\mathrm{Fe} / \mathrm{H}]=-1.7$, $-1.6, \ldots,-1.0,-0.5,-0.25,0\}$ parameter space (with $[\mathrm{Fe} / \mathrm{H}] \geq-1.3$ in the case of $17 \mathrm{Gyr}$ due to model limitations) and a set of composite populations where the previously defined populations were mixed at the 10, 20 and 30\% level (in mass) with a second generation of 5 Gyr old stars with solar metallicity. The solid lines are the best least squares fits to the two branches exhibit by the 116 data points. The parabolic branch stretching from $0.85<(B-R)<1.35$ is solely defined by single-burst, old $(>12 \mathrm{Gyr})$, metal-poor $([\mathrm{Fe} / \mathrm{H}]<-0.5)$ stellar populations. Slightly younger (8-12 Gyr), more metal-rich populations or populations with a second burst of star formation fall onto a linear branch from $1.0<(B-R)<1.5$. A colour independent offset of $0.13 \mathrm{mag}$ was applied to the model data to match the empirical zero point (see text).

distribution showed also some evidence for a minor subclumb of galaxies around NGC 1316 with velocities $\approx 100 \mathrm{~km} \mathrm{~s}^{-1}$ higher than the average. However, due to the small number of galaxies involved the effect on the overall mean cluster velocity was minimal $\left(15 \mathrm{~km} \mathrm{~s}^{-1}\right)$ and well within the $1 \sigma$ uncertainty. Thus, we used the velocity from the complete sample given above for the following calculation.

The conversion of the observed cluster velocity to the frame of the cosmic microwave background, essentially required two corrections: a correction of $-115 \pm 18 \mathrm{~km} \mathrm{~s}^{-1}$ for the solar
Table 8. Distances of the dwarf ellipticals and the Fornax cluster.

\begin{tabular}{|c|c|c|c|c|}
\hline $\begin{array}{c}\text { Galaxy } \\
\text { (1) }\end{array}$ & $\begin{array}{l}\text { Type } \\
\text { (2) }\end{array}$ & $\begin{array}{c}(m-M)_{0} \\
(\mathrm{mag}) \\
(3)\end{array}$ & $\begin{array}{c}\text { Random error } \\
\text { (mag) } \\
\text { (4) }\end{array}$ & $\begin{array}{c}\text { Method } \\
\text { (5) }\end{array}$ \\
\hline FCC043 & $\mathrm{dS} 0, \mathrm{~N}$ & $31.66 \pm 0.11$ & 0.05 & SBF(R) \\
\hline FCC050 & $\mathrm{dE} 0, \mathrm{~N}$ & $31.55 \pm 0.28$ & 0.26 & $\mathrm{SBF}(\mathrm{R})$ \\
\hline FCC082 & $\mathrm{dE} 1, \mathrm{~N}$ & $31.82 \pm 0.36$ & 0.35 & $\operatorname{SBF}(\mathrm{R})$ \\
\hline FCC085 & $\mathrm{dE} 0, \mathrm{~N}$ & $31.37 \pm 0.18$ & 0.15 & $\mathrm{SBF}(\mathrm{R})$ \\
\hline FCC 100 & $\mathrm{dE} 4, \mathrm{~N}$ & $31.84 \pm 0.16$ & 0.12 & $\mathrm{SBF}(\mathrm{R})$ \\
\hline FCC116 & $\mathrm{dE} 1, \mathrm{~N}$ & $31.30 \pm 0.19$ & 0.16 & $\mathrm{SBF}(\mathrm{R})$ \\
\hline FCC136 & $\mathrm{dE} 2, \mathrm{~N}$ & $31.55 \pm 0.14$ & 0.10 & $\mathrm{SBF}(\mathrm{R})$ \\
\hline FCC 150 & $\mathrm{dE} 4, \mathrm{~N}$ & $31.31 \pm 0.16$ & 0.13 & $\mathrm{SBF}(\mathrm{R})$ \\
\hline mean & & $31.54 \pm 0.07$ & & \\
\hline
\end{tabular}

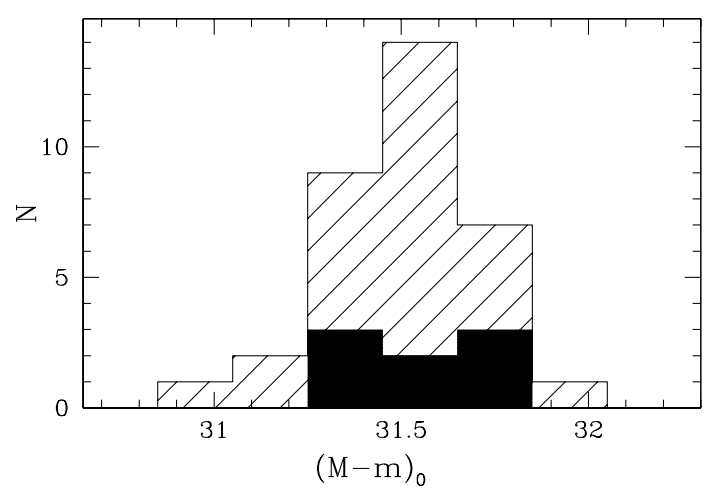

Fig. 9. Distribution of the SBF distance moduli of 33 early-type galaxies in the Fornax cluster. The solid part of the histogram are the dE,Ns while the shaded part are the giants. Galaxies are binned in intervals of $0.2 \mathrm{mag}$.

motion relative to the barycenter of the Local Group (Courteau \& van den Bergh 1999) and a projected Virgocentric flow correction of $-54 \pm 9 \mathrm{~km} \mathrm{~s}^{-1}$ in the direction of Fornax. The latter is based on the distance geometry and a $1 / R$ flow-field characteristics of the Local Group-Virgo-Fornax system as outlined in Madore et al. (1999) and the assumption of an infall velocity of $240 \pm 40 \mathrm{~km} \mathrm{~s}^{-1}$ of the Local Group into the Virgo cluster (Jerjen \& Tamman 1993) which is in good agreement with $224 \pm 90 \mathrm{~km} \mathrm{~s}^{-1}$ (Bureau et al. 1996) and $220 \pm$ $50 \mathrm{~km} \mathrm{~s}^{-1}$ (Tammann \& Sandage 1985). The resulting cosmological expansion velocity $v_{\mathrm{CMB}}^{\text {Fornax }}=1324 \pm 41 \mathrm{~km} \mathrm{~s}^{-1}$ was then divided by the Fornax cluster distance of $20.3 \pm 0.7 \mathrm{Mpc}$ to obtain the Hubble constant of $65 \pm 4 \mathrm{~km} \mathrm{~s}^{-1} \mathrm{Mpc}^{-1}$. This result is in excellent agreement with the more robust value of $69 \pm 5 \mathrm{~km} \mathrm{~s}^{-1} \mathrm{Mpc}^{-1}$ established from a sample of 24 galaxy clusters extending to $9000 \mathrm{~km} \mathrm{~s}^{-1}$ (Giovanelli et al. 1997) and is also consistent at $95 \%$ confidence with both the most recent result of $59 \pm 6 \mathrm{~km} \mathrm{~s}^{-1} \mathrm{Mpc}^{-1}$ from the team that favours a long distance scale (Saha et al. 2001) and the final value of $72 \pm 8 \mathrm{~km} \mathrm{~s}^{-1} \mathrm{Mpc}^{-1}$ adopted by the HST "Key Project" team (Freedman et al. 2001).

\section{On the ages and metallicities of the cluster dEs}

The Worthey+Padova models shown in Fig. 8 predict a change in the $R$-band fluctuation luminosity depending on age and metallicity of the underlying stellar population. Single-burst, 
Table 9. Distance moduli for other Fornax galaxies.

\begin{tabular}{cccc}
\hline \hline & & $(m-M)_{0}$ & Method \\
Galaxy & Type & $(\mathrm{mag})$ & \\
$(1)$ & $(2)$ & $(3)$ & $(4)$ \\
\hline IC1919 & dS0,N & $31.31 \pm 0.17$ & SBF(I) \\
NGC 1316 & S0 & $31.66 \pm 0.17$ & SBF(I) \\
NGC 1336 & E & $31.38 \pm 0.21$ & SBF(I) \\
E358-006 & S0,N & $31.35 \pm 0.32$ & SBF(I) \\
NGC 1339 & E & $31.61 \pm 0.35$ & SBF(I) \\
NGC 1344 & E & $31.48 \pm 0.30$ & SBF(I) \\
NGC 1351 & E & $31.61 \pm 0.16$ & SBF(I) \\
NGC 1366 & S0 & $31.62 \pm 0.29$ & SBF(I) \\
NGC 1373 & E & $31.78 \pm 0.74$ & SBF(I) \\
NGC 1375 & S0 & $31.58 \pm 0.13$ & SBF(I) \\
NGC 1374 & E & $31.48 \pm 0.13$ & SBF(I) \\
E358-025 & S0/a & $31.25 \pm 0.26$ & SBF(I) \\
NGC 1379 & E & $31.51 \pm 0.15$ & SBF(I) \\
NGC 1380 & S0/a & $31.32 \pm 0.18$ & SBF(I) \\
NGC 1381 & S0 & $31.28 \pm 0.21$ & SBF(I) \\
NGC 1386 & Sa & $31.09 \pm 0.25$ & SBF(I) \\
NGC 1380A & S0 & $31.00 \pm 0.29$ & SBF(I) \\
NGC 1387 & SB0 & $31.54 \pm 0.26$ & SBF(I) \\
NGC 1382 & SB0 & $31.77 \pm 0.31$ & SBF(I) \\
NGC 1389 & SB0 & $31.68 \pm 0.18$ & SBF(I) \\
NGC 1399 & E & $31.50 \pm 0.16$ & SBF(I) \\
NGC 1404 & E & $31.61 \pm 0.19$ & SBF(I) \\
NGC 1419 & E & $31.42 \pm 0.24$ & SBF(I) \\
NGC 1427 & E & $31.86 \pm 0.24$ & SBF(I) \\
E358-059 & E & $31.60 \pm 0.20$ & SBF(I) \\
IC2006 & E & $31.59 \pm 0.29$ & SBF(I) \\
\hline mean & & $31.50 \pm 0.04$ & \\
\hline NGC 1326A & SBc & $31.43 \pm 0.07$ & Cepheids \\
NGC 1365 & SBbc & $31.50 \pm 0.10$ & Cepheids \\
NGC 1425 & Sb & $31.93 \pm 0.06$ & Cepheids \\
\hline mean & & $31.62 \pm 0.16$ & \\
\hline & & &
\end{tabular}

old, and metal-poor stellar populations have fainter and bluer $R$-band fluctuations (i.e. data on the parabolic branch) than more metal-rich and/or composite single-burst populations with a fraction of intermediate-age stars (ie. data on the linear branch). The effects on age and metallicity are largely degenerate, making it generally impossible to draw conclusions from changes in fluctuation. However, the simple finding that the studied Fornax dEs (with arguably one exception) have their data on the lower part of the linear branch strongly suggests galaxy ages younger than $12 \mathrm{Gyr}$. This upper limit is consistent with the mean age of $10 \pm 1$ Gyr derived for other Fornax cluster dEs by Rakos et al. (2001) from age sensitive narrow band photometry in the modified Strömgren filtersystem.

Furthermore, the plot symbols along the linear branch in Fig. 8 indicate that this part of the colour-fluctuation luminosity relation is primarily a metallicity sequence. As differences in age cause only a relative small change in $(B-R)_{0}$, the colours of the SBF fields may offer a rough but interesting way to estimate the metallicity of our early-type dwarf galaxies. We
Table 10. $M_{V}$ and metallicity range predicted from $(B-R)$ colours of the SBF fields.

\begin{tabular}{ccccc}
\hline \hline Galaxy & $\begin{array}{c}M_{V} \\
(\mathrm{mag})\end{array}$ & $\begin{array}{c}{[\mathrm{Fe} / \mathrm{H}]} \\
(\text { Minimum })\end{array}$ & $\begin{array}{c}{[\mathrm{Fe} / \mathrm{H}]} \\
(\text { Median })\end{array}$ & $\begin{array}{c}{[\mathrm{Fe} / \mathrm{H}]} \\
(\text { Maximum })\end{array}$ \\
\hline FCC043 & -18.88 & -1.0 & $\mathbf{- 0 . 9}$ & -0.5 \\
FCC050 & -15.66 & -1.6 & $\mathbf{- 1 . 5}$ & -1.5 \\
FCC082 & -16.14 & -1.5 & $\mathbf{- 1 . 4}$ & -1.4 \\
FCC085 & -15.89 & -1.4 & $-\mathbf{1 . 0}$ & -0.7 \\
FCC100 & -17.08 & -1.5 & $-\mathbf{1 . 4}$ & -1.0 \\
FCC116 & -15.93 & -1.0 & $-\mathbf{1 . 0}$ & -1.0 \\
FCC136 & -17.55 & -1.5 & $-\mathbf{1 . 0}$ & -0.9 \\
FCC150 & -16.33 & -1.4 & $\mathbf{- 1 . 0}$ & -0.8 \\
\hline
\end{tabular}

calculated median metallicity and metallicity spread for each dwarf via a direct comparison of the colour range covered by the SBF fields with the corresponding metallicity range shown in Fig. 8. The results are listed in Table 10. The dwarf ellipticals have estimated $[\mathrm{Fe} / \mathrm{H}]$ values ranging from -1.6 to -0.5 .

To test these results we have two possibilities. Firstly, Held \& Mould (1994) measured metallicities of $10 \mathrm{dEs}$ in Fornax from spectral line indices. They found similar values for the three galaxies that we have in common: $[\mathrm{Fe} / \mathrm{H}]_{\mathrm{FCC} 085}=-0.90 \pm$ $0.38,[\mathrm{Fe} / \mathrm{H}]_{\mathrm{FCC} 0100}=-1.28 \pm 0.38$, and $[\mathrm{Fe} / \mathrm{H}]_{\mathrm{FCC} 085}=-0.87 \pm$ 0.05 .

A second, more qualitative test is provided by the predictions of the empirical relation between absolute visual magnitude and $[\mathrm{Fe} / \mathrm{H}]$. The relation wherein fainter galaxies have lower $[\mathrm{Fe} / \mathrm{H}]$ was studied for 15 low luminous dEs and dwarf spheroidals in the Local Group by Da Costa (1998) and is reproduced in Fig. 10. Dwarfs with luminosities in the magnitude range from $M_{V}=-9$ to -16 mag helped to define the relation. More luminous dEs like the sample studied by Held \& Mould (1994) are unique to the cluster environment (Ferguson \& Binggeli 1994). We show this second data set as triangles in Fig. 10. For the conversion of the provided $V$ magnitudes to luminosities we either adopted our Fornax cluster distance modulus of 31.54 or used the measured SBF distance if available.

It is now interesting to see where our bright nucleated dEs are located in this graph. For that purpose, we calculated in Col. (2) of Table 10 the absolute $V$ magnitudes from the $B$ magnitudes in Table 1, employing the newly derived distance moduli (Col. (3) of Table 8) and using a typical $B-V=0.72$ or the measured $B-V$ values (Bothun et al. 1989). We plotted in Fig. 10 (filled circles) the median metallicities as derived from the $(B-R)$ colours versus $M_{V}$ for our dEs. The error bars simply indicate the estimated metallicity range.

Although the scatter among the Held \& Mould dEs is larger than the scatter observed for the LG dEs, our dwarf ellipticals lie in a region of the diagram that indicates they are an extension of the relation. There is a hint that 3-4 dEs (including FCC100 whose metallicity was confirmed by Held \& Mould) are slightly too metal-poor for their total luminosity. Such a deviation is understandable in terms of an age effect. According to the finding of Rakos et al. (2001) dEs younger than $10 \mathrm{Gyr}$ 


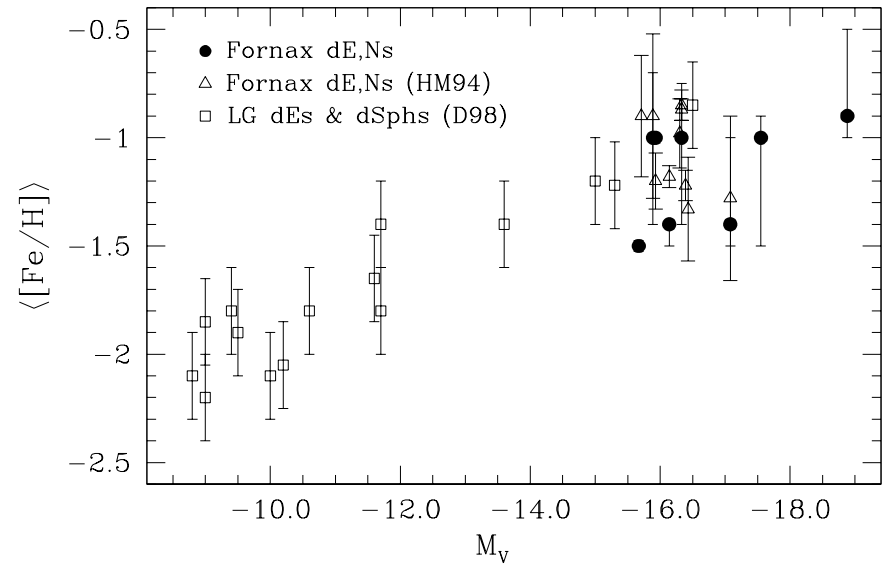

Fig. 10. The median metallicities plotted as a function of absolute $V$-band luminosity for our sample Fornax dE,Ns (filled circles). The error bars indicate the metallicity range estimated from the colour range of the SBF fields. The open triangles and squares are the corresponding data for a sample of Fornax dEs (Held \& Mould 1994) and the dEs and dSphs in the Local Group (da Costa 1998). Here error bars are taken from the respective reference.

tend to lie above the metallicity-luminosity relation and the dEs with ages greater than 10 Gyr below. This suggest ages of $10 \mathrm{Gyr}$ or older for our dEs. If combined with the previously found upper age limit we conclude that they must have ages in the range between 10 and 12 Gyr. Alternatively, Barazza \& Binggeli (2002) have suggested that the scatter in the metallicity-luminosity relation for bright $\mathrm{dEs}$ is in large part due to differences in ellipticity, rather than age as claimed by Rakos et al. (2001). With our small sample we cannot confirm, nor reject this ellipticity effect. However, we like to point out that roundness was one of our sample selection criteria (see Sect. 1) leading to a narrow ellipticity range of $0.05<\epsilon<0.25$ for our dwarfs.

The two performed consistency checks found good agreements with independent and more accurate data and thus underlines the usefulness of the $(B-R)$ colour as rough metallicity indicator for bright dEs. But it needs to be recalled that for the method to work it is required to known that the galaxy occupies the linear branch of the colour-fluctuation luminosity relation.

The fact that all eight, arguably FCC116, Fornax dEs are on the linear branch stands somehow in contrast to the six nearby dEs studied by Jerjen et al. (2001) with data on the parabolic branch. An interpretation in terms of the stellar content of these galaxies suggests that the dEs from the dense cluster environment are younger (10-12 Gyr) than their cousins populating the lower density environments (M81 group, Canes Venatici I cloud). In addition, the latter dwarfs appear as single-burst stellar systems whereas bright cluster dEs have the clear signature of a second, intermediate-age component. These observational differences may be taken as indicator for the importance of the global environmental density for the evolution of $\mathrm{dE}$ galaxies.

\section{Summary}

We have reported on the first application of the surface brightness fluctuation method to measure accurate distances to earlytype $d$ warf galaxies in the Fornax cluster. Deep $B$ and $R$-band images of eight nucleated dwarf ellipticals have been analysed to measure extinction-corrected $(B-R)$ colours and $R$-band SBF magnitudes $\bar{m}_{R}$ for 92 fields distributed over the central regions of the galaxies. For all dwarfs but one, a strong linear relation between the two quantities was found in accordance with the predictions of stellar population synthesis models. This allowed the use of the semi-empirical equation $\bar{M}_{R}=6.09 \cdot(B-R)_{0}-8.94$ (Jerjen et al. 2001) to calibrate the $\left[(B-R)_{0}, \bar{m}_{R}^{0}\right]$ data and to determine the SBF distances of the galaxies. All sample dEs that were previously associated to Fornax only by means of redshift and/or morphology could be confirmed as cluster members. The SBF distances are determined to $\sim 9 \%$. The distribution of the dwarfs centered well at a distance of $(m-M)_{0}=31.54 \pm 0.07 \mathrm{mag}$ or $20.3 \pm 0.7 \mathrm{Mpc}$, a value that is fully consistent with recent work on Fornax cluster ellipticals and thus appears as the currently best estimate for the Fornax cluster distance.

It has been more difficult to make secure claims on the line-of-sight depth of the cluster. We have estimated the quantity from our data using bootstrap techniques. The preliminary value of $1.4_{-0.8}^{+0.5} \mathrm{Mpc}$ suggested a spatially compact galaxy aggregate that is possibly slightly more extended along the line of sight. However, this result is preliminary at the current stage and needs to be confirmed. Improved statistics can be expected from the analysis of the complete program sample of 25 Fornax $\mathrm{dEs}$ in the near future.

We combined the best cosmological velocity of the Fornax cluster with the newly derived Fornax modulus from the dEs to obtain a Hubble constant of $H_{0}=65 \pm 4 \mathrm{~km} \mathrm{~s}^{-1} \mathrm{Mpc}^{-1}$. The value is in excellent agreement with more robust measurements and statistically conform with the most recent result by Saha et al. (2001) and the final value adopted by the Hubble Space Telescope Key Project team (Freedman et al. 2001).

These first results on distances of Fornax dEs shed further light on the great potential of the SBF method to study large numbers of early-type dwarf galaxies in the local Universe in an efficient way. Among others, this opens up the perspective of dynamical studies of galaxy cluster core regions by means of measuring distances and redshifts of entire $\mathrm{dE}$ populations.

As an experiment, we also tested the $(B-R)$ as possible metallicity estimator for bright dEs. This idea is based on the fact that for composite single-burst old stellar populations the relation between $(B-R)_{0}$ colour and fluctuation luminosity represents primarily a metallicity sequence. Using this method, we derived median $[\mathrm{Fe} / \mathrm{H}]$ values in the range from -1.5 to -1 with a concentration around $[\mathrm{Fe} / \mathrm{H}]=-1$. The good agreement with more precise spectroscopic $[\mathrm{Fe} / \mathrm{H}]$ measurements published in the literature was encouraging. The colour-based metallicities also moved the Fornax dEs right to the extension of metallicity-luminosity relation that was originally defined by the intrinsically much fainter Local Group dEs. The data further suggests an age range between 10 and 12 Gyr for our Fornax dEs.

Acknowledgements. I wish to thank Bruno Binggeli and the referee Dr Steven Phillipps for carefully reading the manuscript and for helpful suggestions. I am grateful to the anonymous ESO service observer at the VLT UT3 for obtaining the excellent quality images that were required for this work. 


\section{References}

Barrazza, F. D., \& Binggeli, B. 2002, A\&A, 394, L15

Barazza, F. D., Binggeli, B., \& Jerjen, H., 2002, A\&A, 391, 809

Bellazzini, M., Ferraro, F. R., \& Pancino, E. 2001, ApJ, 556, 635

Bertelli, G., Bessan, A., Chiosi, C., Fagotto, F., \& Nasi, E. 1994, A\&AS, 106, 271

Binggeli, B., Tammann, G. A., \& Sandage, A. 1987, AJ, 94, 251

Binggeli, B., \& Cameron, L. M. 1991, A\&A, 252, 27

Binggeli, B., \& Jerjen, H. 1998, A\&A, 333, 17

Bothun, G. D., Schombert, J. M., \& Caldwell, N. 1989, AJ, 98, 1542

Bureau, M., Mould, J. R., \& Staveley-Smith, L. 1996, ApJ, 463, 60

Courteau, S., \& van den Bergh, S. 1999, AJ, 118, 337

Cowie, L. L., Gardner, J. P., Hu, E. M., et al. 1994, ApJ, 434, 114

Da Costa, G. S. 1998, Stellar astrophysics for the local group: VIII Canary Islands Winter School of Astrophysics, ed. A. Aparicio, A. Herrero, \& F. Sanchez (Cambridge; New York: Cambridge University Press), 351

Da Costa, G. S., \& Armandroff, T. A. 1990, AJ, 100, 162

da Costa, L. N., Willmer, C. N. A., Pellegrini, P. S., et al. 1998, AJ, 116,1

de Jong, R. S. 1996, A\&A, 313, 377

Drinkwater, M. J., Gregg, M. D., \& Colless, M. 2001, ApJ, 548, L139

Efron, B., \& Tibshirani, R. J. 1986, Statist. Science, 1, 54

Ferguson, H. C. 1989, AJ, 98, 367

Ferrarese, L., Mould, J. R., Kennicutt, R. C. Jr., et al. 2000, ApJ, 529, 745

Ferguson, H. C., \& Binggeli, B. 1994, A\&RA, 6, 67

Freedman, W. L., Madore, B. F., Gibson, B. K., et al. 2001, ApJ, 553, 47

Giovanelli, R., Haynes, M. P., da Costa, L. N., et al. 1997, ApJ, 477, L1

Grebel, E. K. 1998, in IAU Symp. 192, The Stellar Content of Local Group Galaxies, ed. P. Whitelock, \& R. Cannon (San Francisco: ASP), 1

Hanuschik, R., \& Silva, D. 2002, The Messenger, 108, 4

Harris, W. E., Durrell, P. R., Pierce, M. J., \& Secker, J. 1998, Nature, 395,45

Held, E. V., \& Mould, J. R. 1994, AJ, 107, 1307

Jensen, J. B., Tonry, J. L., \& Luppino, G. A. 1998, ApJ, 505, 111
Jerjen, H., \& Binggeli, B. 1997, Are dwarf ellipticals genuine ellipticals?, Proceedings of the Second Stromlo Symposium on The Nature of Elliptical Galaxies, ed. M. Arnaboldi et al., ASP Conf. Ser., 116, 239

Jerjen, H., \& Dressler, A. 1997, A\&AS, 124, 1

Jerjen, H., \& Tammann, G. A. 1993, A\&A, 1993, 276, 1

Jerjen, H., Binggeli, B., \& Barazza, F. D. 2003, in preparation

Jerjen, H., Freeman, K. C., \& Binggeli, B. 1998, AJ, 115, 1840

Jerjen, H., Freeman, K. C., \& Binggeli, B. 2000, AJ, 116, 166

Jerjen, H., Kalnajs, A., \& Binggeli, B. 2000, A\&A, 358, 845

Jerjen, H., Rekola, R., Takalo, L., Coleman, M., \& Valtonen, M. 2001, A\&A, 380, 90

Karachentsev, I. D., Makarov, D. I., \& Huchtmeier, W. K. 1999, A\&AS, 139, 97

Karachentsev, I. D., Sharina, E., Grebel, E., et al. 2000, ApJ, 542, 128

Kelson, D. D., Illingworth, G. D., Tonry, J. L., et al. 2000, ApJ, 529, 768

Landolt, A. U. 1992, AJ, 104, 340

Madore, B. F., Freedman, W. L., Silbermann, N., et al. 1999, ApJ, 515, 29

Miller, B. W., Lotz, J. M., Ferguson, H. C., Stiavelli, M., \& Whitmore, B. C. 1998, ApJ, 508, L133

Mould, J. R., Hughes, S. M. G., Stetson, P. B., et al. 2000, ApJ, 528, 655

Phillipps, S., Drinkwater, M. J., Gregg, M. D., \& Jones, J. B. 2001, 560, 201

Prosser, C. F., Kennicutt, R. C. Jr., Bresolin, F., et al. 1999, ApJ, 525, 80

Rakos, K., Schombert, J., Maitzen, H. M., Prugovecki, S., \& Odell, A. 2001, AJ, 121, 1974

Saha, A., Sandage, A., Tammann, G. A., et al. 2001, ApJ, 562, 314

Schlegel, D., Finkbeiner, D., \& Davis, M. 1998, ApJ, 500, 525

Silbermann, N., Harding, P., Ferrarese, L., et al. 1999, ApJ, 515, 1

Stein, P. 2002, private communication

Stetson, P. B. 1987, PASP, 99, 191

Tammann, G. A., \& Sandage, A. 1985, ApJ, 294, 81

Tonry, J. L., Dressler, A., Blakeslee, J. P., et al. 2001, ApJ, 546, 681

Tonry, J. L., \& Schneider, D. P. 1988, AJ, 96, 807

Worthey, G. 1994, ApJS, 95, 107 